THE EURO

OVERNIGHT

INTERBANK

MARKET AND

ECB'S LIQUIDITY

MANAGEMENT

POLICY DURING

TRANQUIL AND

TURBULENT TIMES

by Nuno Cassola

and Michael Huetl 


\title{
THE EURO OVERNIGHT INTERBANK MARKET AND ECB'S LIQUIDITY MANAGEMENT POLICY DURING TRANQUIL AND TURBULENT TIMES'
}

\author{
by Nuno Cassola ${ }^{2}$ and Michael Huet ${ }^{3}$
}

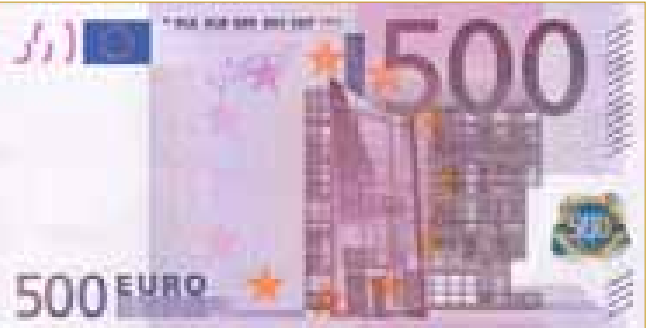

In 2010 all ECB

publications

feature a motif

taken from the

$€ 500$ banknote.

NOTE: This Working Paper should not be reported as representing the views of the European Central Bank (ECB).

The views expressed are those of the authors and do not necessarily reflect those of the $E C B$.

This paper can be downloaded without charge from http://www.ecb.europa.eu or from the Social Science Research Network electronic library at http://ssrn.com/abstract_id=1678446.

I This paper was presented to the ECB Workshop "Challenges to Monetary Policy Implementation beyond the Financial Market Turbulence", Frankfurt am Main, Germany on 01//2/2009. Comments provided by an anonymous referee, by Gunther Wuyts and participants

to the workshop are gratefully acknowledged. We thank Hugo Rodríguez Mendizábal for generously providing the simulation source code applied in [GQM08].

2 European Central Bank, Kaiserstrasse 29,603II Frankfurt am Main, Germany; e-mail: nuno.cassola@ecb.europa.eu

3 University of St. Gallen, Swiss Institute of Banking and Finance, Rosenbergstrasse 52, 9000 St. Gallen, 
(C) European Central Bank, 2010

Address

Kaiserstrasse 29

60311 Frankfurt am Main, Germany

Postal address

Postfach 160319

60066 Frankfurt am Main, Germany

Telephone

+496913440

Internet

http://www.ecb.europa.eu

Fax

+496913446000

All rights reserved.

Any reproduction, publication and reprint in the form of a different publication, whether printed or produced electronically, in whole or in part, is permitted only with the explicit written authorisation of the ECB or the author(s).

Information on all of the papers published in the ECB Working Paper Series can be found on the ECB's website, http://www. ecb.europa.eu/pub/scientific/wps/date/ html/index.en.html

ISSN 1725-2806 (online) 


\section{CONTENTS}

Abstract

Non-technical summary

1 Introduction

2 Operational framework and stylised facts

2.1 Main features of the operational framework

2.2 Stylized facts

3 The [QM06] model of banks' liquidity management

4 Regime-switching model of reserve management by banks

5 Fine-tuning operation on $t=T$ and excess reserves

5.1 Excess reserves

5.2 Fine-tuning operation

6 Modeling the impact of the financial crisis

6.1 Market segmentation

6.2 Aggregate liquidity shock

6.3 Lending constraints

7 How does frontloading work?

8 Calibration of the model parameters

8.1 Timing of the regime-switch

8.2 Simulations

9 Policy evaluation

10 Conclusions

References

Figures and tables 


\begin{abstract}
We analyze the impact of the recent financial market crisis on the Euro Overnight Index Average (EONIA) and interbank market trading and assess the effectiveness of the ECB liquidity policy between 07/2007 - 08/2008. We extend the model of [QM06] by (i) incorporating the microstructure of the EONIA market including the ECB fine-tuning operation on the last day of the maintenance period (MP) and banks' daily excess liquidity, (ii) giving insight into banks' trading behavior characterized by an endogenous regime-switch and suggesting an efficient procedure to simulate the entire MP, and (iii) proposing a model for market distortion due to lending constraints which lead to a bid-ask spread for the EONIA rate. The model is calibrated by simulation fitting daily EONIA rates and aggregate liquidity measures observed between March 2004 and September 2008. Besides lending constraints we consider market segmentation and aggregate liquidity shocks as possible market distortions in the crisis period. For a calibration cross-check and for estimating the timing of the endogenous regime-switch we use panel data covering liquidity data of 82 Euro Area commercial banks for the period 03/2003 - 07/2007. With the calibrated model the ECB policy of liquidity frontloading is evaluated and compared with a reserve band system policy similar to the Bank of England's framework. We find that liquidity frontloading is a small scale central bank intervention which is capable of stabilizing interest rates in both frictionless and distorted markets. Simulations suggest that without frontloading the EONIA would have been, on average, 23 basis points above the policy rate (target); with frontloading, the overnight rate is, on average, on target.
\end{abstract}

Keywords: liquidity management, open market operations, simulation, microstructure JEL: E44, E52, G21 


\section{NON-TECHNICAL SUMMARY}

This paper develops a theoretical framework to analyze the impact of the financial market crisis on the euro overnight interbank market, and to assess the effectiveness of the liquidity policy followed by the European Central Bank (ECB) during the August 2007 - September 2008 period. The paper has two main parts.

In the first part we extend the seminal work of Quirós and Mendizábal (2006) in three dimensions. First, we generalize the model by casting it into a regime-switching model in which banks change liquidity management from a backward-looking liquidity shock correction mode into a forward-looking reserve management regime. Second, we explicitly incorporate the microstructure of the EONIA interbank market in our analysis. We model (i) the ECB policy of fine-tuning operations (i.e. provision or absorbtion of liquidity to mitigate liquidity imbalances) on the last day of the RMP, (ii) daily excess reserves held by banks as observed in aggregate liquidity data and (iii) weekend effect which seems to be rather influential during the financial turmoil and cause the EONIA to be U-shaped during the last week of the RMP. Consequently, we are able to explain and reproduce the empirically observed level and shape of the EONIA rates in the last week of the maintenance period. Third, in order to analyse the impact of the financial crisis on the euro interbank market we consider three types of market distortions: (1) credit rationing; (2) market segmentation; and (3) aggregate (negative) liquidity shocks.

In the second part of the paper we calibrate the theoretical model and conduct simulation exercises in order to evaluate the impact of the liquidity policies of the ECB.

The main conclusions from calibration are as follows: (1) market segmentation and lending constraints were not a significant feature of the Euro inter-bank overnight market before the crisis; (2) increasing liquidity volatility (idiosyncratic or aggregate) is neither necessary nor sufficient to generate the kind of market developments that were observed during the crisis; (3) to replicate the trading patterns observed during turmoil one needs a combination of increasing market segmentation and credit limits (rationing).

With the calibrated model, the ECB's liquidity frontloading policy is evaluated and compared with a reserve band system policy similar to the Bank of England's framework (which we model following MacGorian (2005 a, b). Simulations suggest that without frontloading the overnight interest rate would have been, on average, 23 basis points above the policy rate (target); with frontloading and a fine-tuning operation on the last day of the maintenance period, the overnight rate is, on average, on target. Comparing the fine-tuning policy of the ECB to a reserve band system similar to the framework of the Bank of England we find that in case of liquidity frontloading both systems are equally able to anchor the overnight rate on the last day of the maintenance period where the latter seems to improve over the former in terms of higher average interbank market trading, lower transaction costs and lower EONIA standard deviation.

There is a caveat in our analysis that should be borne in mind. With its liquidity policy the central bank fosters market activity and steers the overnight interest rate; however, the source of market distortions, captured in the model by market segmentation and lending constraints, is not addressed/impacted by the policy (i.e. are kept as exogenous parameters in the simulations). This can be justified only if those distortions have their roots in solvency, rather than liquidity problems. 


\section{Introduction}

The main goal of this paper is to develop a theoretical framework to analyze the impact of the financial market crisis on the euro overnight interbank market, and assess the effectiveness of the liquidity policy followed by the European Central Bank (ECB) during the August 2007 September 2008 period.

Whilst the unsecured euro interbank money market for maturities above one-week was severely disrupted by the financial market turmoil since its beginning, with vanishing volumes and large spreads over secured sources of funding (e.g. the repo market), the unsecured overnight interbank market saw an increase in transactions volumes, relatively low spreads and volatility in the overnight interest rate (EONIA) ${ }^{1}$. Therefore, it is unclear whether market frictions were pervasive (and if this was the case of what type) in the euro interbank market during turmoil.

The liquidity policy followed by the ECB during the August 2007 - September 2008 period, labeled frontloading, consisted of shifting the timing of the supply of refinancing, within the reserve maintenance period, rather than changing the overall (average) size of funds available to the banking system. Thus, it is somewhat puzzling how the ECB managed to steer the overnight interest rate so close to the policy rate under turmoil (at an average zero spread) given the very limited costs in terms of increased overnight interest rate volatility and frequency of fine-tuning operations. At that time, some commentators referred to the policy as "flooding the market with liquidity", which is a misinterpretation of frontloading. This paper attempts to shed light on the question of how frontloading works.

Our theoretical framework is based on the seminal work of Quirós and Mendizábal (2006) (herein [QM06]). In their framework banks are assumed to receive a liquidity shock at the end of the day after the market for daily funds has closed (see Poole (1968) [Poo68]). Facing this uncertainty about end-of-day liquidity shocks, banks manage their reserves by trading on the interbank market so that the costs of borrowing (lending) liquidity shortages (surpluses) from (to) the ECB are minimized.

In the first part of the paper we extend [QM06] in several dimensions. Firstly, we generalize the [QM06] model by casting it into a regime-switching model in which banks change liquidity management from a backward-looking liquidity shock correction mode into a forward-looking reserve management regime; in the model, banks follow the former mode in the early stages of the reserve maintenance period (RMP) and switch to the latter mode towards its end, the

\footnotetext{
${ }^{1}$ EONIA is a quantity weighted average of the interest rate charged on overnight loans by a panel of Euro area banks.
} 
timing of the switch being endogenously determined. With this adjustment we are not forced to limit our simulations to the 3 last trading days as in Gaspar, Quirós and Mendizábal (2008) (herein [GQM08]) to tackle the problem of curse of dimensionality but we are able to simulate the entire maintenance period of approximately 28 days.

Secondly, we explicitly incorporate the microstructure of the EONIA interbank market in our analysis. We model (i) the ECB policy of fine-tuning operations (i.e. provision or absorbtion of liquidity to mitigate liquidity imbalances) on the last day of the RMP, (ii) daily excess reserves held by banks as observed in aggregate liquidity data and (iii) weekend effect which seems to be rather influential during the financial turmoil and cause the EONIA to be U-shaped during the last week of the RMP. Consequently, we are able to explain and reproduce the empirically observed level and shape of the EONIA rates in the last week of the maintenance period.

Thirdly, in order to analyse the impact of the financial crisis on the euro interbank market we consider three types of market distortions: 1) credit rationing, 2) market segmentation as implemented in [GQM08], and 3) aggregate (negative) liquidity shocks. The assumptions underlying our model of credit rationing are motivated by the observation of increased banks' overnight lending to the ECB. We assume that banks prefer the ECB as counterparty and only partly lend to the interbank market. Thereby we show that banks' lending constraints lead to a bid-ask spread for the interbank market rate.

In the second part of the paper we calibrate the theoretical model and conduct simulation exercises in order to evaluate the impact of the liquidity policies of the ECB. The model is calibrated by a systematic search for a combination of parameters that minimize the Euclidian distance of simulated versus realised values of the interest rate and liquidity aggregates. The simulations are carried out along the lines suggested in [GQM08] and are conditional on aggregate liquidity data observed between March 2004 and September 2008. For cross-checking the calibration of the idiosyncratic liquidity shock volatility and for estimating the timing of the regime-switch we use GMM methods and survival analysis, respectively, using individual bank's liquidity data collected for 82 Euro Area commercial banks covering the period March 2003 July 2007.

The main conclusions from calibration are as follows: 1) market segmentation and lending constraints were not a significant feature of the Euro inter-bank overnight market before the crisis; 2) increasing liquidity volatility (idiosyncratic or aggregate) is neither necessary nor sufficient to generate the kind of market developments that were observed during the crisis; 3) to replicate the trading patterns observed during turmoil one needs a combination of increasing 
market segmentation and credit limits (rationing).

With the calibrated model, the ECB's liquidity frontloading policy is evaluated and compared with a reserve band system policy similar to the Bank of England's framework (which we model following MacGorian (2005 a, b) [Mac05b] and [Mac05a]). Simulations suggest that without frontloading the overnight interest rate would have been, on average, 23 basis points above the policy rate (target); with frontloading and a fine-tuning operation on the last day of the maintenance period, the overnight rate is, on average, on target. Comparing the fine-tuning policy of the ECB to a reserve band system similar to the framework of the Bank of England we find that in case of liquidity frontloading both systems are equally able to anchor the overnight rate on the last day of the maintenance period where the latter seems to improve over the former in terms of higher average interbank market trading, lower transaction costs and lower EONIA standard deviation. However, if we assume that liquidity imbalances are not mitigated by frontloading in the early stage of the maintenance period, compared to the fine-tuning operation system, the reserve band system is less effective in steering interest rates towards the policy target rate.

There is a caveat in our analysis that should be borne in mind. With its liquidity policy the central bank fosters market activity and steers the overnight interest rate; however, the source of market distortions, captured in the model by market segmentation and lending constraints, is not addressed / impacted by the policy (i.e. are kept as exogenous parameters in the simulations). This can be justified only if those distortions have their roots in solvency, rather than liquidity problems.

The remainder of the paper is structured as follows. Section 2 reviews the main features of the operational framework of the Eurosystem and presents stylized facts about liquidity management by banks in the euro area, the euro overnight interbank market and the open market operations of the ECB, before and after August 2007. The empirical evidence provides the motivation for our theoretical framework. Section 3 reviews the [QM06] model and introduces the notation. In Section 4 we present the regime-switching model. In Section 5 we adapt the model of [QM06] to incorporate banks' demand for excess reserves and the ECB's fine-tuning operation on the last day of the maintenance period. In Section 6 we discuss aggregate liquidity shocks and introduce lending constraints in the model. The principles of frontloading are discussed in Section 7. Model calibration is presented in Section 8 including the estimation of the timing of the regime switch and the distribution of individual bank's liquidity shocks; we discuss alternative scenarios based on simulating different combinations of liquidity shocks, lending constraints and 
market segmentation both for the pre-crisis and crisis periods and compare the simulation results with observed aggregate values. In Section 9 we simulate and evaluate different open market operations policies and different degrees of frontloading. The main conclusions are presented in Section 10

\section{Operational Framework and Stylised facts}

\subsection{Main features of the operational framework}

The operational framework of the Eurosystem contains three main elements: minimum reserve requirements with averaging provision, standing facilities, and open market operations. Below we briefly describe each of these components.

Reserve requirements contribute to stabilizing money market interest rates. Euro area banks have to keep minimum reserves (ie current accounts) with National Central Banks (NCB). Reserves must be kept on average over a maintenance period which has approximately one month duration. Required reserves are remunerated - linked to the marginal rate of the Main Refinancing Operations (MROs). Current account holdings beyond the minimum requirement are not remunerated (excess reserves).

Additionally, there are two types of standing facilities, one providing liquidity (against collateral), which is the marginal lending facility and another, absorbing liquidity, which is the deposit facility. Both are overnight facilities taken at the discretion of the banks, and, in general, there are no limits set by the ECB to their recourses by banks. Standing facilities have penalty rates: marginal lending +100 basis points above the Minimum Bid Rate (MBR, policy rate) and deposit facility -100 basis points below the MBR. These two rates set a corridor for the interbank market overnight interest rate.

There are three main types of open market operations:

1. The Main Refinancing Operations (MROs) are liquidity providing reverse transactions, with one-week maturity, conducted every week.

2. The Longer Term Refinancing Operations (LTROs) are liquidity providing reverse transactions, with three-month maturity, conducted once a month. ${ }^{2}$

3. Fine Tuning Operations (FTOs) provide or absorb liquidity. They have neither fixed

\footnotetext{
${ }^{2}$ During the crisis the ECB introduced other term refinancing operations: Supplementary Longer Term Refinancing Operations (SLTRO) with 3-month, 6-month and 12-month maturities; and a Special Term Refinancing Operation (Maintenance Period Operation - MPO) with maturity equal to the length of the reserve maintenance period, with allotment and settlement on its first day.
} 
frequency nor maturity. Since 2005 the ECB conducts (almost) systematically an FTO on the last day of each reserve maintenance period.

\subsection{Stylized Facts}

This section presents stylised facts about banks' liquidity management in the euro area, the euro overnight interbank market and the open market operations of the ECB, before and after August $2007 .^{3}$ The aim of the section is to provide empirical motivation for the theoretical framework and the extensions to the [QM06] model that will be introduced in the following sections.

Our sample of daily aggregate data includes both tranquil and turbulent times. The beginning of the pre-crisis sample is March 2004, which coincides with the introduction of several changes to the operational framework of the Eurosystem. One change relevant for the modelling framework is the execution of fine-tuning operations on the last day of the maintenance period, i.e. the provision or absorbtion of liquidity to mitigate liquidity imbalances. While the framework for FTOs was setup in March 2004, these operations have been carried out with higher frequency since February 2005 (see [ECB06]). To the extent that fine-tuning operations are anticipated by market participants the overnight interest rate is expected to settle close to the key policy rate ${ }^{4}$ on the last day of the reserve maintenance period. However, demand for excess reserves by (some) banks introduces an asymmetry in the fine-tuning operation which makes its implementation complicated. The following sections will deal with this difficulty.

The crisis period in our sample starts on 9 August 2007, when the French bank BNP Paribas closed three investment funds with exposure to US sub-prime loans. The sample ends on 15 September 2008, when the investment bank Lehman Brothers collapsed and the ECB switched to fixed rate tenders with full allotment. The analysis of the second "wave" of financial market turbulence is outside the scope of the present paper. The pre-crisis (crisis) period covers 33 (14) maintenance periods.

Open market operations During the crisis period the ECB followed a frontloading liquidity policy, i.e. additional liquidity was provided via allotments above benchmark ${ }^{5}$ during the early stage of the reserve maintenance period with the surplus gradually reduced throughout the reserve maintenance period, either through allotments below benchmark or via liquidity draining fine-tuning operations.

\footnotetext{
${ }^{3}$ Further information on the operational framework of the Eurosystem is presented in the Appendix.

${ }^{4}$ The key policy rate of the ECB is the minimum bid rate in the weekly Main Refinancing Operations.

${ }^{5}$ Benchmark allotment is defined as the amount of refinancing that allows banks to fulfil their reserve requirements smoothly (linearly) over the reserve maintenance period.
} 
Figure 5 plots the average outstanding volumes of refinancing operations over the last 28 days of the reserve maintenance period, normalised by the minimum reserve requirement, and the empirical distribution of fine-tuning operation volumes on the last day of the reserve maintenance period. In the pre-crisis period the provision of liquidity via MROs and LTROs was stable throughout the reserve maintenance period; the outstanding refinancing via MROs was higher than the outstanding longer term refinancing via LTROs (see Figure 5a, b). There were no FTOs except on the last day of the maintenance period (see Figure 5c); on the last day of the maintenance period FTO volumes were very small and showed a symmetrical distribution centered around zero (see Figure 5d). In total there were 11 liquidity providing FTOs and 14 liquidity absorbing FTOs.

In the crisis period liquidity frontloading was implemented by high and decreasing tender amounts at the MROs. At the same time, and in contrast to the pre-crisis period, the share of liquidity provision via MROs became lower than the share of liquidity provision via LTROs; there was a shift towards longer-term refinancing by the ECB in order to fill the funding gaps of banks that were opened by the drying-up of market term funding after the crisis (see Figure 5 e, f). During the crisis FTOs were conducted also in the early part of the reserve maintenance period and were on average liquidity draining operations (see Figure $5 \mathrm{~g}$ ). On the last day of the maintenance period liquidity has been drained by fine-tuning operations with volumes centered around $-10 \%$ of the MRR (see Figure $5 \mathrm{~h}$ ). Hence, the ECB's open market operations policy in the crisis period can be characterized by liquidity frontloading through MROs during the entire maintenance period and liquidity absorbtion via FTOs during the reserve maintenance period.

Current accounts and reserve fulfilment path by banks Figure 6 shows evidence on banks' current accounts and information on their reserve fulfilment path over the last 28 days in the maintenance period. In the pre-crisis period banks fulfilled their reserve requirement in a smooth way; the deficiency ${ }^{6}$ was linearly reduced over the maintenance period and the current accounts were stable, slightly above $100 \%$ of the minimum reserve requirements reflecting average excess reserves of approximately 5-8 bps per MRR (see Figure 6a, b). Daily liquidity conditions were stable throughout the maintenance period with only minor daily imbalances emerging (Figure 6c).

In the crisis period the deficiency deviated from the linear fulfilment path due to the frontloading policy of the ECB. In fact, banks' current accounts decreased on average from 110\%

\footnotetext{
${ }^{6}$ Deficiency at a certain day is the remaining reserve requirement to be met until the end of the maintenance period.
} 
of MRR at the beginning of the maintenance period down to $80 \%$ of the MRR at the end of the maintenance period (Figure 6e). The deficiency decreased faster in the early stage of the maintenance period compared to the pre-crisis period (Figure 6f). As a result, daily liquidity conditions varied significantly throughout the maintenance period with sizable daily imbalances emerging (Figure 6g). The mean aggregate accumulated reserve surplus on the last day of the maintenance period was non-zero and centered around $14.5 \%(12 \%)$ of the MRR in the pre-crisis (crisis) period.

EONIA Figure 7 plots the average EONIA and the total interbank market trading volume over the last 28 days of the maintenance period in the pre-crisis and the crisis period. In the precrisis period, EONIA stayed almost constant at approximately $7-8$ bps above the MBR most of the time with increasing volatility towards the end of the maintenance period; on the last day of the maintenance period EONIA converged towards the MBR level (Figure 7a) thus suggesting a small deviation from the strict martingale hypothesis. In the pre-crisis period interbank market trading volume exhibited a marked increasing pattern over the maintenance period (Figure 7c).

In the crisis period interbank market rates show higher volatility with the EONIA spread (over the MBR) moving around zero. Around weekends and towards the last days of the maintenance period EONIA showed a marked U-shape pattern with a minimum on the penultimate day (Figure $7 \mathrm{~b}$ ). Comparing the trading volume of pre-crisis and crisis period one observes a slightly higher (lower) trading volume in the crisis period at the beginning (end) of the maintenance period reflecting again the frontloading policy of the ECB (Figure $7 \mathrm{~d}$ ).

Standing facilities Figure 8 plots the recourses to the marginal lending facility (MLF) and the deposit facility (DF). During the crisis period banks' recourses to the deposit facility were higher than in the pre-crisis period (Figure 8a, b), while the recourses to the marginal lending facility remained approximately unchanged or were even lower during the crisis period (Figure 8c, d).

Modelling challenges posed by the empirical evidence It is noticeable that in both the pre-crisis and in the crisis period the EONIA is close to the MBR on the last day of the maintenance period; however, it shows a slight declining trend during the maintenance period, and a U-shape pattern towards its end. Simultaneously we observe an aggregate accumulated reserve surplus of $14.5 \%$ (12\%) of the MRR and mean FTO liquidity absorbtion of $0 \%(10 \%)$ of the MRR on the last day of the maintenance period in the pre-crisis (crisis) period. These 
observations cannot be explained by the [QM06] model.

Moreover, neither [QM06] nor [GQM08] allow for lending constraints which was a widespread feature of the interbank market during the crisis period, as reported by banks. Further and possibly related to the issue of lending constraints, the [QM06]/[GQM08] framework does not take into account the empirical observation that banks' lending to the ECB deposit facility increased in the crisis period, while banks' borrowing from the ECB lending facility remained unchanged.

In the following sections we present a theoretical framework capable of explaining the stylized facts and incorporating a variety of market distortions.

\section{The [QM06] model of banks' liquidity management}

In this section we review the seminal [QM06] model of the market for daily funds in the Euro area. The model incorporates the ECB standing facilities which are an important feature of the Eurosystem operational framework and provides the backbone for our own model.

The model considers $n$ banks and assumes that the interbank market is frictionless. A bank $j$ at time $t$ is characterized by the tuple $\left(a_{t}^{j}, d_{t}^{j}\right)$ where $a_{t}^{j}$ denotes the bank's current account kept at the central bank and $d_{t}^{j}$ is the deficiency, i.e. the remaining reserve requirement on day $t$ of the maintenance period. Each bank trades the amount $b_{t}^{j}$ in the interbank market, where $b_{t}^{j}<0\left(b_{t}^{j}>0\right)$ corresponds to borrowing (lending) funds. After the interbank market closes each bank $j$ receives a liquidity shock $\lambda_{t}^{j}$, which we interpret as resulting from imperfect monitoring of outflows and inflows of funds. The liquidity shocks are assumed to follow a probability distribution $F$; more specifically we consider a normal distribution with mean $\mu$ and standard deviation $\sigma$, i.e. $\lambda_{t}^{j} \sim N(\mu, \sigma)$. The $j$-th bank's current account and deficiency are updated every day such that

$$
a_{t+1}^{j}=a_{t}^{j}+\lambda_{t}^{j}, \quad d_{t+1}^{j}=\max \left(d_{t}^{j}-\max \left(a_{t+1}^{j}-b_{t}^{j}, 0\right), 0\right),
$$

respectively (see Figure 1). A bank must fulfil its reserve requirement on average over a maintenance period; therefore $d_{T+1}^{j}=0$, where $T$ denotes the last day of the maintenance period. 


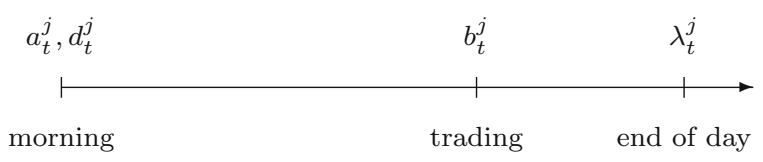

Figure 1: Intra-day schedule

In the [QM06] model the interbank market rate is determined by the costs of using the central bank's standing facilities, i.e. the marginal lending rate $i^{l}$ and the deposit rate $i^{d}$ for borrowing and lending funds from and to the central bank, respectively.

Overnight overdrafts are not allowed by the central bank; thus, if a bank faces an overdraft on days prior to the last day of the maintenance period, $t<T$, i.e. if $a_{t+1}^{j}-b_{t}^{j}<0$, it has to borrow from the marginal lending facility of the central bank at the marginal lending rate $i^{l}$. If a bank fulfills its reserve requirement before the end of the maintenance period, i.e. $d_{t}^{j}-a_{t+1}^{j}+b_{t}^{j}=0$, it is said to be locked-in. In this case the bank lends the surplus to the central bank at the deposit rate $i^{d}$. On the last day $t=T$ of the maintenance period the bank must borrow from the marginal lending facility any unfulfilled reserve requirement $d_{T}^{j}-\left(a_{T}^{j}+\lambda_{T}^{j}-b_{T}^{j}\right)$ at rate $i^{l}$. If it is locked-in, it lends to the central bank at the deposit rate $i^{d}$. Given that, compared to interbank market trading, transacting with the central bank involves penalty rates, i.e. it is costly for a bank to fulfil its reserve requirement ahead of time or to miss it on the last day.

On the last day of the maintenance period, $t=T$, the solution of the model gives the optimal trading $b_{T}^{j}$ and market clearing rate $i_{T}$ which are equal to, respectively

$$
b_{T}^{j}=D_{T}-A_{T}-\left(d_{T}^{j}-a_{T}^{j}\right), \quad i_{T}=i^{d}+\left(i^{l}-i^{d}\right) F\left(D_{T}-A_{T}\right)
$$

where $D_{t}=\frac{\sum_{j=1}^{n} d_{t}^{j}}{n}$ and $A_{t}=\frac{\sum_{j=1}^{n} a_{t}^{j}}{n}$ are the aggregate deficiency per bank and the aggregate current account per bank on day $t$, respectively (see [QM06]).

Liquidity conditions are said to be balanced on the last day of the reserve maintenance period, when $D_{T}=A_{T}$. If $F()$ is symmetric around zero, i.e. $\lambda_{t}^{j} \sim N(0, \sigma)$, balanced liquidity conditions, $F(0)=1 / 2$, imply that, $i_{T}=i^{d}+\left(i^{l}-i^{d}\right) / 2$, i.e. the equilibrium level of the overnight interest rate is the mid-point of the interest rate corridor set by the rates on the standing facilities $\left(i^{l}\right.$ and $\left.i^{d}\right)$. Therefore, in order to steer the overnight interest rate the central bank may choose to set the rates on the standing facilities symmetrically around the policy rate $\left(i^{*}\right)$ given that the market equilibrium and the "targeted" policy levels of the overnight interest rate would coincide $\left(i_{T}=i^{*}\right)$, at least on the last day of the reserve maintenance period, provided that it ends with 
balanced liquidity conditions.

For days $t<T-1$ prior to the penultimate day of the maintenance period there is no closed form solution to the liquidity management problem. The bank determines the borrowing or lending such that

$$
i_{t}^{j}=i^{l} F\left(b_{t}^{j}-a_{t}^{j}\right)+i^{d}\left(1-F\left(d_{t}^{j}+b_{t}^{j}-a_{t}^{j}\right)\right)-\int_{b_{t}^{j}-a_{t}^{j}}^{d_{t}^{j}+b_{t}^{j}-a_{t}^{j}} \frac{\partial V_{t+1}}{\partial d_{t+1}^{j}} f\left(\lambda_{t}^{j}\right) d \lambda_{t}^{j} .
$$

where

$$
\frac{\partial V_{t}}{\partial d_{t}^{j}}=\left\{\begin{array}{ll}
-i_{T} & \text { if } t=T \\
-i^{d}\left(1-F\left(d_{t}^{j}+b_{t}^{j}-a_{t}^{j}\right)\right)+\int_{-\infty}^{d_{t}^{j}+b_{t}^{j}-a_{t}^{j}} \frac{\partial V_{t+1}}{\partial d_{t+1}^{j}} f\left(\lambda_{t}^{j}\right) d \lambda_{t}^{j} & \text { if } t<T
\end{array} .\right.
$$

The intuition for equation 3 is as follows: In equilibrium, banks equate the marginal revenue of lending funds ( $i_{t}^{j}$, the market rate) to the expected marginal cost. The expected marginal cost is a weighted average of three terms: the cost of marginal lending, which happens with probability $F\left(b_{t}^{j}-a_{t}^{j}\right)$ which may happen if, for example, the bank borrows "too little" in the market and then faces a "large" end-of-day liquidity shock $\left(\lambda_{t}^{j}-b_{t}^{j}+a_{t}^{j}<0\right)$; the cost of being locked-in, which happens with probability $1-F\left(d_{t}^{j}+b_{t}^{j}-a_{t}^{j}\right)$, which may occur if, for example, the bank borrows "too much" in the market and then faces a "small" end-of-day liquidity shock $\left(d_{t}^{j}-a_{t}^{j}-\lambda_{t}^{j}+b_{t}^{j}=0\right)$; and the cost of carrying one unit of reserve deficiency into the future, which is measured by the third term on the r.h.s. of equation 3. Further intuition for this dynamic cost term can be gained by looking at the solution for the penultimate day in the reserve maintenance period, $T-1$.

As shown in [QM06], the rate $i_{t}^{j}$ on days $t<T$ can be approximated by

$$
i_{t}^{j}=i^{l} F\left(b_{t}^{j}-a_{t}^{j}\right)+i^{d}\left(1-F\left(d_{t}^{j}+b_{t}^{j}-a_{t}^{j}\right)\right)+E\left[i_{t+1}\right] \int_{b_{t}^{j}-a_{t}^{j}}^{d_{t}^{j}+b_{t}^{j}-a_{t}^{j}} f\left(\lambda_{t}\right) d \lambda_{t} .
$$

On day $T-1$ equation (5) becomes

$$
\begin{aligned}
i_{T-1}^{j}= & i^{l} F\left(b_{T-1}^{j}-a_{T-1}^{j}\right)+i^{d}\left(1-F\left(d_{T-1}^{j}+b_{T-1}^{j}-a_{T-1}^{j}\right)\right) \\
& +E\left[i_{T}\right]\left(F\left(d_{T-1}^{j}+b_{T-1}^{j}-a_{T-1}^{j}\right)-F\left(b_{T-1}^{j}-a_{T-1}^{j}\right)\right)
\end{aligned}
$$

According to equation (6) the equilibrium overnight rate on the penultimate day of the reserve maintenance period $(T-1)$ is a weighted average of three rates: $i^{l}$ with weight equal to the probability of an overdraft on that day, $F\left(b_{T-1}^{j}-a_{T-1}^{j}\right) ; i^{d}$ with weight equal to the probability of the bank locking-in on that day, $1-F\left(d_{T-1}^{j}+b_{T-1}^{j}-a_{T-1}^{j}\right)$; and $E\left[i_{T}\right]$, the 
expected rate on the last day of the maintenance period, with a weight equal to the probability that the bank is neither locked-in nor faces an overdraft on day $T-1$. Thus, the cost of carrying one unit of reserve deficiency into the last day of the reserve maintenance period is measured by $E\left[i_{T}\right]$, conditional on the bank being neither locked-in nor face an overdraft on day $T-1$.

In the special case when, $F\left(d_{T-1}^{j}+b_{T-1}^{j}-a_{T-1}^{j}\right)-F\left(b_{T-1}^{j}-a_{T-1}^{j}\right)=1$, then $i_{T-1}^{j}=E\left[i_{T}\right]$; if balanced liquidity conditions are expected for day $T, E\left[i_{T}\right]=i^{*}$, and then, $i_{T-1}^{j}=i^{*}$; under these special conditions the central bank is able to steer the overnight interest rate at the policy rate level also on the penultimate day of the reserve maintenance period. However, these conditions are, in practice, difficult to verify and may not extend backwards in time before $T-1$, as it will be elaborated in the following sections.

\section{Regime-switching model of reserve management by banks}

In this section we introduce the main building blocks of our theoretical framework. We start with the observation that there is no analytical solution to the model of [QM06] for all but the last two days of the reserve maintenance period. Therefore, solutions for days $t<T-1$ have to be obtained through numerical approximation techniques as done also in [GQM08]. Still, simulation of a single maintenance period with more than three days is hard to get without further simplifications. In fact, solving the model for maintenance periods of 28 days, like the average length of a reserve maintenance period in the euro area, is too time consuming. Thus, in the early stages of the reserve maintenance period, treasurers at commercial banks are unlikely to manage their daily trading in the interbank market by taking the entire reserve maintenance period into account.

As a simplifying assumption [QM06] suggest to impose the martingale property for the overnight interest rate in the model, which simplifies the simulations:

$$
i_{t}=E\left[i_{t+1}\right]
$$

However, assuming the martingale property during the early stage of the reserve maintenance period leaves the distribution of interbank market trading $b_{t}^{j}$ indeterminate. This, of course, is not an appealing feature of the model especially if one is interested in explaining trading volumes. In fact, even if the indeterminacy of lending volumes might be acceptable during tranquil times, during turmoil the distribution of trading volumes becomes an important driver of interbank market dynamics. Our modelling approach allows pinning-down both rates and volumes and, thus, is suitable for analysing the interbank market under both calm and turbulent conditions. 
We simplify equation (6) by noting that, given the size of the reserve requirement in the euro area, at the beginning of the maintenance period the probability of a bank getting locked-in is virtually zero,

$$
1-F\left(d_{t}^{j}+b_{t}^{j}-a_{t}^{j}\right) \approx 0 .
$$

Thus, from equation (6) and equation (8) we see that in the early stages of the reserve maintenance period a bank determines its interbank market trading according to

$$
i_{t}^{j}=E\left[i_{t+1}\right]+\left(i^{l}-E\left[i_{t+1}\right]\right) F\left(b_{t}^{j}-a_{t}^{j}\right)
$$

i.e. the rate $i_{t}^{j}$ depends on the expected rate $i_{t+1}$ for the next day and the probability $F\left(b_{t}^{j}-a_{t}^{j}\right)$ of having an overdraft; it is not dependent on the deficiency. Thus, management of no-overdrafts can be considered as the primary reason for interbank market trading in the early stages of the reserve maintenance period.

Rearranging equation (9) gives the optimal interbank market trading $b_{t}^{j}$ of the $j$-th bank,

$$
b_{t}^{j}=a_{t}^{j}+F^{-1}\left(\frac{i_{t}^{j}-E\left[i_{t+1}\right]}{i^{l}-E\left[i_{t+1}\right]}\right) .
$$

If the interbank market is cleared at the rate $i_{t}^{j}=i_{t}$, then

$$
0=\sum_{j=1}^{n} b_{t}^{j}=\sum_{j=1}^{n} a_{t}^{j}+n F^{-1}\left(\frac{i_{t}-E\left[i_{t+1}\right]}{i^{l}-E\left[i_{t+1}\right]}\right) .
$$

If $i_{t}^{j}=i_{t}$ and from equation (10) and equation (11) the supply of funds $b_{t}^{j}$ can be calculated as

$$
b_{t}^{j}=-A_{t}+a_{t}^{j} .
$$

Therefore in the early stage of the maintenance period a bank trades the deviation of its current account $a_{t}^{j}$ from the market's average current account $A_{t}$. If the bank's previous current account is at about the level of the current market's average, i.e. if $A_{t} \approx a_{t-1}^{j}$, the bank will borrow funds if it received a negative shock on the previous day and will lend if a positive shock was received, i.e. the bank follows an error (liquidity shock) correction mechanism. Furthermore, if shocks are assumed to sum up to zero across banks on each day in the maintenance period,

$$
\sum_{j=1}^{n} \lambda_{t-1}^{j}=0
$$

and if the market is frictionless, then

$$
\begin{aligned}
b_{t}^{j} & =-\frac{\sum_{j=1}^{n} a_{t-1}^{j}}{n}+a_{t-1}^{j}+\lambda_{t-1}^{j} \\
& =-\frac{\sum_{j=1}^{n} a_{t-2}^{j}}{n}+a_{t-2}^{j}+\lambda_{t-1}^{j}+\lambda_{t-2}^{j} \\
& =\sum_{s=1}^{t-1} \lambda_{s}^{j}
\end{aligned}
$$


where $a_{1}^{j}=a_{1}$; in a frictionless market the bank's interbank borrowing or lending is independent of the liquidity shocks of the other banks and only depends on the own shocks received in the past.

If the fund supply is given by equation (12), the deficiency is updated by

$$
d_{t}^{j}=\max \left(d_{t-1}^{j}-\max \left(A_{t-1}+\lambda_{t-1}^{j}, 0\right), 0\right)
$$

which means that the deficiency is reduced by the average current account in the market $A_{t-1}$ and the own shock $\lambda_{t-1}^{j}$ experienced on the previous day.

Inserting equation (12) into equation (9) gives the equilibrium rate which is equal to

$$
i_{t}=E\left[i_{t+1}\right]+\left(i^{l}-E\left[i_{t+1}\right]\right) F\left(-A_{t}\right) .
$$

The equilibrium overnight rate depends only on the average current account $A_{t}$ and the expected rate on the next day $E\left[i_{t+1}\right]$. Note that as the market rate is bounded from above by the marginal facility rate, $i^{l}$, the second term on the right hand side of equation (18) must be non-negative, which suggests that the martingale hypothesis might not be verified in the early stages of the reserve maintenance period; in fact the overnight rate might be expected to decline over the reserve maintenance period $\left(i_{t} \geq E\left[i_{t+1}\right]\right)$, which is consistent with the evidence presented in Section 2.

Compared to the models [QM06] and [GQM08], we differentiate between two regimes. Regime $I$ represents the early stage of the maintenance period when the probability of getting locked-in is zero and the backward-looking liquidity shock correction model described in this section applies. Under this first regime, banks only manage overdrafts and overlook the deficiency. Under the first regime rates and trading are determined by equations (9) and (12), respectively. However, as soon as the probability of getting locked-in crosses a certain threshold, $1-F\left(d_{t}^{j}+b_{t}^{j}-a_{t}^{j}\right)>\epsilon$, a switch takes place into regime $I I$ where funds supplied $b_{t}^{j}$ and the optimal overnight interest rate $i_{t}^{j}$ are determined by the forward-looking equations proposed by [QM06] and reviewed in Section 3. The timing of the regime switch will be discussed in Section 8 .

\section{$5 \quad$ Fine-tuning operation on $t=T$ and excess reserves}

In the model of [QM06] banks expect the overnight rate to be equal to the policy rate on the last day of the maintenance period if liquidity is balanced, i.e. if average deficiency is equal to the average current account. However, in Section 2 which reports stylized facts in the Euro area we observe rates near the policy rate along with liquidity surpluses indicating that the banking system accumulates excess reserves of about $14.5 \%(12.0 \%)$ of the daily average reserve 
requirement in the pre-crisis (crisis) period without increasing interbank market lending pressure on the last day of the maintenance period. Moreover, starting in February 2005 with higher frequency, the ECB carries out a fine-tuning operation on the last day of the maintenance period, which mitigates liquidity deviations from the seemingly targeted excess reserves. Both targeted excess reserves and fine-tuning operations are not modelled in [QM06]. We address this shortcoming by explicitly taking into account the ECB's fine-tuning operation $f$ on the last day of the maintenance period, $t=T$, and the targeted (accumulated) excess reserves, $S$ of banks.

\subsection{Excess Reserves}

Excess reserves are not remunerated and therefore should be zero given the alternative offered to banks to place any surplus at the deposit facility, which is remunerated. However, in the Eurosystem recourse to the deposit facility is not automatic; it must be requested by the bank. The empirical fact that there are excess reserves in the euro area can be explained if the opportunity cost of holding a (small) liquidity surplus is lower than the marginal cost of paying the treasurer to transfer excess funds to the deposit facility (see [BCMHW06]). For small institutions, which have low reserve requirements, this may indeed be the case.

Each bank's liquidity surplus on $t=T$ after interbank market trading and receiving shocks is given by

$$
a_{T}^{j}-b_{T}^{j}-d_{T}^{j}+\lambda_{T}^{j}
$$

As $\sum_{j=1}^{n} b_{T}^{j}=0$, the per bank aggregate liquidity surplus is

$$
\sum_{j=1}^{n} a_{T}^{j}-b_{T}^{j}-d_{T}^{j}+\lambda_{T}^{j}=A_{T}-D_{T}
$$

Based on the argument in [BCMHW06] we assume that banks keep daily excess reserves which accumulate up to the average excess reserves $S$ on the last day of the maintenance period. Furthermore, we assume that the bank trades $b_{t}^{j}-X$ in the interbank market, i.e. banks trade the additional amount $-X$ to keep daily excess reserves as buffer. In this case it can be shown in analogy to the derivation in [QM06] that the equilibrium interbank market rate on the last day of the maintenance period is given by

$$
i_{T}=i^{d}+\left(i^{l}-i^{d}\right) F\left(S-D_{T}+A_{T}\right)
$$

where on the last day $X$ does not enter the equation as no overdrafts are managed on this day. For days $t<T$ prior to the last day of the maintenance period the formulas for the $i$-th bank's 
optimal rate are given by

$$
i_{t}^{j}=i^{l} F\left(b_{t}^{j}-a_{t}^{j}-X\right)+i^{d}\left(1-F\left(S+d_{t}^{j}+b_{t}^{j}-a_{t}^{j}-X\right)\right)-\int_{b_{t}^{j}-a_{t}^{j}-X}^{S+d_{t}^{j}+b_{t}^{j}-a_{t}^{j}-X} \frac{\partial V_{t+1}}{\partial d_{t+1}^{j}} f\left(\lambda_{t}^{j}\right) d \lambda_{t}^{j} .
$$

where

$$
\frac{\partial V_{t}}{\partial d_{t}^{j}}=\left\{\begin{array}{ll}
-i_{T} & \text { if } t=T \\
-i^{d}\left(1-F\left(S+d_{t}^{j}+b_{t}^{j}-a_{t}^{j}-X\right)\right)+\int_{-\infty}^{d_{t}^{j}+b_{t}^{j}-a_{t}^{j}-X} \frac{\partial V_{t+1}}{\partial d_{t+1}^{j}} f\left(\lambda_{t}^{j}\right) d \lambda_{t}^{j} & \text { if } t<T
\end{array} .\right.
$$

Correspondingly the interbank market trading in regime I is given by

$$
b_{t}^{j}=-A_{t}+a_{t}^{j}+X
$$

(cf equation (12)). Hence, if $X<0$ banks borrow more and lend less in order to achieve excess liquidity. For the remaining theoretical discussion we assume $X=0$ in regime I and only consider the impact of targeted excess reserves on the last day of the maintenance period.

\subsection{Fine-tuning operation}

According to equation (2) interbank market rates increase with decreasing liquidity surplus. To mitigate such effects of liquidity imbalances on interbank market rates the ECB carries out a fine-tuning operation on the last day of the reserve maintenance period, when the averaging mechanism ceases to apply (see Figure 2).

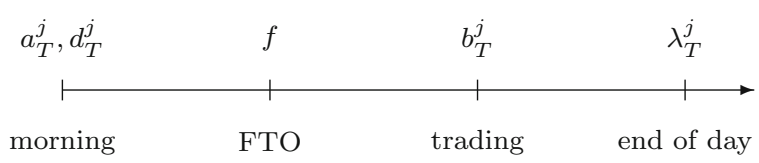

Figure 2: Intra-day schedule on the last day of the maintenance period

We assume that the ECB determines the fine-tuning operation as a function $h$ of the aggregate liquidity surplus $A_{T}-D_{T}$ after trading and recourses to the standing facilities and the accumulated excess reserves $S$ targeted by banks on $t=T$, i.e.

$$
f=h\left(A_{T}, D_{T}, S\right)
$$

where we assume that in case of a liquidity surplus $f<0$ and in a liquidly deficit $f>0$. The amount $S$ represents the willingness of banks to keep excess reserves. Hence $S$ is equal to the accumulated excess reserves held per bank during the maintenance period. 
Liquidity is balanced if $f=0$. In case of liquidity imbalances we assume that through the fine-tuning operation $f$ the central bank provides or drains liquidity at the rate $i^{f}$ which we assume to be equal to the MBR. ${ }^{7}$

If $f<0$, i.e. in case of a liquidity surplus, the $\operatorname{costs} c_{T}^{j}$ of a bank using the ECB's standing facilities at $t=T$ are given by borrowing at the marginal lending facility at the rate $i^{l}$ up to the targeted accumulated excess reserves $S$, i.e. if $a_{T}^{j}-d_{T}^{j}-b_{T}^{j}+\lambda_{T}^{j}<S$, or by lending at the rate $i^{f}$ up to an amount of $-f$ and lending the remaining surplus at the rate $i^{d}$ such that the targeted accumulated excess reserves $S$ are met. The optimal trading $b_{T}^{j}$ is determined by ${ }^{8}$

$$
i_{T}^{j}=i^{d}+\left(i^{l}-i^{f}\right) F\left(d_{T}^{j}+b_{T}^{j}-a_{T}^{j}+S\right)+\left(i^{f}-i^{d}\right) F\left(d_{T}^{j}+b_{T}^{j}-a_{T}^{j}+S-f\right) .
$$

In case of a liquidity deficit, i.e. if $f>0$, the $\operatorname{costs} c_{T}^{j}$ are calculated as the cost of borrowing, where up to the amount $f$ the rate $i^{f}$ applies and for the remaining amount of borrowing the rate $i^{l}$ has to be paid. The costs of lending are given by the rate $i^{d}$. Both borrowing and lending banks are assumed to target accumulated excess reserves of $S$. The corresponding first order condition for the optimal interbank market trading $b_{T}^{j}$ is given by

$$
i_{T}^{j}=i^{d}+\left(i^{l}-i^{f}\right) F\left(d_{T}^{j}-f+b_{T}^{j}-a_{T}^{j}+S\right)+\left(i^{f}-i^{d}\right) F\left(d_{T}^{j}+b_{T}^{j}-a_{T}^{j}+S\right) .
$$

If the rate $i^{f}$ is set equal to the MBR, i.e. if $i^{f}=\frac{i^{l}+i^{d}}{2}$, then the interbank rate for both liquidity providing $(f>0)$ and liquidity absorbing $(f<0)$ fine-tuning operations is equal to

$$
i_{T}^{j}=i^{d}+\frac{i^{l}-i^{d}}{2}\left(F\left(d_{T}^{j}-f+b_{T}^{j}-a_{T}^{j}+S\right)+F\left(d_{T}^{j}+b_{T}^{j}-a_{T}^{j}+S\right)\right) .
$$

If $f$ is evenly distributed across all banks, we can derive a formula for the equilibrium trading amount $b_{T}^{j}$ by the following approach. We start from the model of [QM06] which gives the market clearing trading at the rate $i_{T}$ as

$$
b_{T}^{j}=\left(D_{T}-A_{T}\right)-\left(d_{T}^{j}-a_{T}^{j}\right) .
$$

Inserting into equation (28) gives

$$
i_{T}=i_{T}^{j}=i^{d}+\frac{i^{l}-i^{d}}{2}\left(F\left(S-f+D_{T}-A_{T}\right)+F\left(S+D_{T}-A_{T}\right)\right) .
$$

As $f$ and $S$ are equal for all banks, the rate $i_{T}^{j}$ is equal for all banks and therefore equal to the equilibrium rate $i_{T}$. Therefore, the amount of market trading in case of fine-tuning is the same

\footnotetext{
${ }^{7}$ This is a modelling simplification. In practice, the ECB has provided (drained) liquidity in FTOs using variable rate tender procedures with a minimum (maximum) bid rate equal to the policy rate. The marginal rate in FTOs has been close though not equal to the minimum bid rate.

${ }^{8}$ See Appendix Section B for the derivation of this first order condition.
} 
as in the case of no fine-tuning operation. The difference due to fine-tuning is the equilibrium rate which is higher (lower) in case of a liquidity deficit $f>0$ (surplus $f<0$ ).

Moreover, from equation (30) it follows that if the central bank targets an interbank market rate equal to the MBR, i.e. $i_{T}=\frac{i^{d}+i^{l}}{2}$, then the amount of the fine-tuning operation has to be determined such that

$$
F\left(S-f+D_{T}-A_{T}\right)+F\left(S+D_{T}-A_{T}\right)=1 .
$$

By assumption the distribution $F$ fulfills the condition $1-F(x)=F(-x)$; thus we obtain

$$
f=2\left(S+D_{T}-A_{T}\right)
$$

i.e. the central bank has to provide (drain) twice the banks' perceived liquidity surplus (deficit) $S+D_{T}-A_{T}$ in order to reach an interbank market equilibrium rate equal to the MBR on the last day of the maintenance period. Note that when the maintenance period enters its last day with current accounts sufficiently large, i.e. when $S+D_{T}=A_{T}$, the fine-tuning volume is null, $f=0$. The empirical evidence reviewed in Section 2 suggests that this situation corresponded to the average case before the crisis.

Figure 9 illustrates the relation between the fine-tuning operation and the accumulated daily reserve surplus on the last day of the reserve maintenance period.

As an approximation the FTO volume can be calculated as the difference between the accumulated reserve surplus observed in the morning of the last day of the maintenance period (before trading) and the mean accumulated reserve surplus in the evening (after trading) amounting to $S=14.5 \%(S=12 \%)$ of the $M R R$ in the pre-crisis (crisis) period. Considering both liquidity providing and absorbing FTOs, simple OLS regressions for the amount of the fine-tuning operation $f$ give:

$$
f^{\text {pre-crisis }} \approx-0.01741+1.05912\left(S+D_{T}-A_{T}\right)
$$

and

$$
f^{\text {crisis }} \approx-0.00326+0.597753\left(S+D_{T}-A_{T}\right)
$$

for the pre-crisis and crisis period, respectively, where the coefficients are significantly different from zero at the $1 \%$ level. Furthermore, the regression slopes differ significantly from 2 at the $1 \%$ level (see equation (32)) giving empirical evidence that ECB practise of determining the amount of the FTO deviates from the rule derived above. 


\section{Modeling the impact of the financial crisis}

To analyse the impact of the financial market crisis on the functioning of the overnight interbank market, the model presented in the previous sections has to be refined. We follow the strategy of introducing deviations from and/or constraints on optimality. First, we consider a negative aggregate liquidity shock to the supply of funds in the overnight interbank market. The liquidity shock is assumed to follow a distribution $N(\mu, \sigma)$ where $\mu<0$. The impact of the aggregate liquidity shock on interbank market trading and overnight interest rate is discussed in Section 6.2. Second, we introduce constraints on market lending such that banks lend part of their liquidity surpluses to the central bank (see Section 6.3). In both cases, i.e. lending constraints and negative liquidity shocks, we additionally analyze different degrees of market segmentation (Section 6.1) by simulation (see Section 8.2).

Counterpart risk and uncertainty about own funding needs may be two reasons for banks to lend sub-optimally under turmoil. Both factors also may contribute to market segmentation, which may have been reinforced, after the crisis, due to the segmented (national) nature of banking supervision and financial stability responsibilities, thereby potentially hindering the cross-border flow of liquidity.

\subsection{Market segmentation}

We model market segmentation as suggested by [GQM08]. We group the $n$ banks into smaller groups with size $s, n / s$ being the number of bank groups and allow trading among banks only within each group. Consequently, the shocks within a group do not necessarily sum up to zero leading to aggregate shocks $\sum_{j=1}^{s} \lambda_{t}^{j}=\mu_{t}^{s}$ within each group even though $\lambda_{t}^{j} \sim N(0, \sigma)$ and $\sum_{j=1}^{n} \lambda_{t}^{j}=0$. The aggregate group shock $\mu_{t}^{s}$ can be positive or negative leading to lower or higher group rates, respectively. In the overall market the cross-section dispersion of the interbank market rates increases due to market segmentation (see [GQM08]).

\subsection{Aggregate liquidity shock}

In this section we refine the model by introducing aggregate liquidity shocks. Here we consider only regime $I$; the impact of aggregate liquidity shocks in regime II is simulated and discussed in Section 8.2. We denote $\lambda_{t}^{j-}=-\mu+\lambda_{t}^{j 0}$ the liquidity shock with mean $\mu>0$ bank $j$ receives, where $\lambda_{t}^{j 0} \sim N\left(0, \sigma^{j}\right)$ and

$$
\sum_{i=1}^{n} \lambda_{t}^{j 0}=0 \quad \text { and } \quad \sum_{i=1}^{n} \lambda_{t}^{j-}=-n \cdot \mu
$$


If negative aggregate shocks are received by banks, the current accounts update such that $a_{t+1}^{j}=a_{t}^{j}+\lambda_{t}^{j-}$, where

$$
a_{t+1}^{j} \sim N\left(a_{1}^{j}-\mu \cdot t, \sqrt{t} \sigma\right)
$$

and

$$
a_{t+1}^{j}=a_{1}^{j}-\mu \cdot t+\sum_{s=1}^{t} \lambda_{s}^{j 0} .
$$

The per bank aggregate current account is equal to

$$
\frac{\sum_{i=1}^{n} a_{t+1}^{j}}{n}=A_{1}-\mu \cdot t
$$

Inserting into equation (12), the supply of funds in the interbank market and the equilibrium rate in the early stage of the maintenance period, respectively, are given by

$$
b_{t}^{j}=-A_{1}+\mu \cdot(t-1)+a_{1}^{j}-\mu \cdot(t-1)+\sum_{s=1}^{t-1} \lambda_{s}^{j 0}=-A_{1}+a_{1}^{j}+\sum_{s=1}^{t-1} \lambda_{s}^{j 0}
$$

and

$$
i_{t}=E\left[i_{t+1}\right]+\left(i^{l}-E\left[i_{t+1}\right]\right) F\left(-A_{1}+\mu \cdot(t-1)\right)
$$

Comparing equation (18) and equation (40) one observes that as a consequence of a negative aggregate liquidity shock, the equilibrium overnight interest rate will be higher as the probability of an overdraft increases. According to equation (9) the bank trades the amount $b_{t}^{j}$ so that the deviation of its current account from the market average is zero. As all banks experience the aggregate shock, the deviation of a single current account from the market average is not influenced by the aggregate shock. Therefore, the distribution of the trading $b_{t}^{j}$ in the interbank market is not affected by the negative aggregate liquidity shock.

\subsection{Lending constraints}

The introduction of lending constraints in the model is motivated by the empirical observation of successful liquidity absorbing fine-tuning operations in the early stage of the reserve maintenance period and banks' use of the deposit facility after turmoil (see Figure 5 and Figure 8). We assume that banks supply only part of their optimal unconstrained lending to the interbank market and lend the remainder to the ECB by going to the deposit facility and by participating in liquidity absorbing FTOs.

On the last day of the maintenance period $t=T$ we assume that banks with a liquidity surplus lend $b_{T}^{j} l$ with lending constraint $0<l<1$ to the interbank market at the interbank market rate $i_{T}$ and $b_{T}^{j}(1-l)$ to the ECB by taking recourse to the deposit facility at deposit rate 
$i^{d}$. Then first order optimality condition ${ }^{9}$ for interest rates in the fine-tuning operation model is given by

$$
i_{T}^{j}= \begin{cases}i^{d}+\left(i^{l}-i^{d}\right) \frac{F\left(-f+S+d_{T}^{j}+b_{T}^{j}-a_{T}^{j}\right)+F\left(S+d_{T}^{j}+b_{T}^{j}-a_{T}^{j}\right)}{2 l} & b_{T}^{j}>0 \\ i^{d}+\left(i^{l}-i^{d}\right) \frac{F\left(-f+S+d_{T}^{j}+b_{T}^{j}-a_{T}^{j}\right)+F\left(S+d_{T}^{j}+b_{T}^{j}-a_{T}^{j}\right)}{2} & b_{T}^{j}<0\end{cases}
$$

Hence the equilibrium lending interest rate is higher than the equilibrium borrowing rate (discontinuity at $b_{T}^{j}=0$ ) generating a bid-ask spread given by

$$
\operatorname{spread}_{T}^{j}=\left(i^{l}-i^{d}\right) \frac{F\left(-f+S+d_{T}^{j}+b_{T}^{j}-a_{T}^{j}\right)+F\left(S+d_{T}^{j}+b_{T}^{j}-a_{T}^{j}\right)}{2} \frac{1-l}{l}
$$

The spread is positively dependent on the width of the interest rate corridor $i^{l}-i^{d}$; on the volume of the liquidity absorbing fine-tuning operations $f<0$; on the targeted excess reserves $S$ and the fraction of non interbank market lending $1-l$. The optimal interbank market trading $\min \left(b_{T}^{j}, b_{T}^{j} l\right)$ for different levels of lending constraint $l$ as well as the $\operatorname{spread}_{T}^{j}$ is illustrated in an example in Figure 3.

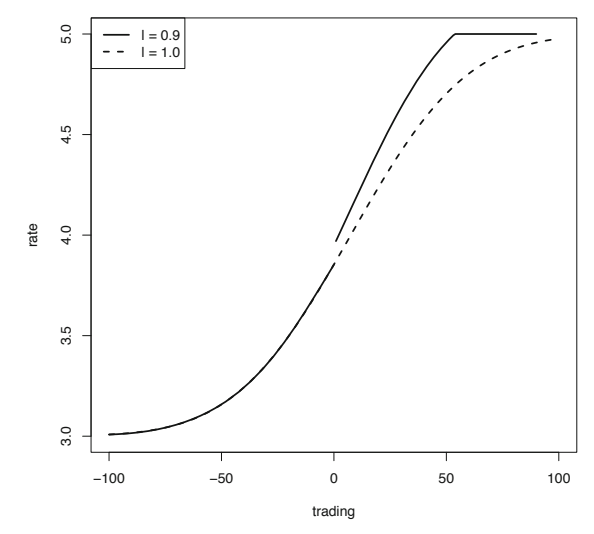

Figure 3: Demand curve for $i^{d}=3 \%, i^{l}=5 \%, D_{T}^{j}=100, A_{T}^{j}=100, f=0, S=0, \lambda_{T}^{j} \sim$ $N(0,40)$.

On days $t<T$, we assume that the banks lend $b_{T}^{j} l$ to the interbank market at rate $i^{T}$ and the remaining lending $b_{T}^{j}(1-l)$ to the ECB at a rate equal to the weighted average of the FTO rate and the deposit rate $i^{d}$. As a proxy we assume that this average is equal to the interbank

\footnotetext{
${ }^{9}$ The profit of a lending bank $j$ on the last day is given by
}

$$
i_{T}^{j} b_{T}^{j} l+i^{d} b_{T}^{j}(1-l)-c(T)
$$

where $c(T)$ are the costs of using the standing facilities in case of an overdraft or in case of getting locked-in (see Section 3 and Section 5). The optimal borrowing/lending $b_{T}^{j}$ is given by

$$
i_{T}^{j}=i^{d}-\frac{i^{d}}{l}+\frac{\partial c(T)}{\partial b_{T}^{j}} .
$$


market rate, which can be observed to fluctuate around the MBR in the crisis period. The equilibrium interbank market trading is given by ${ }^{10}$

$$
\min \left(b_{t}^{j}, b_{t}^{j} l\right)= \begin{cases}\frac{\left(-A_{t}+a_{t}^{j}\right)\left(1+l^{*}\right)+\frac{l^{*}}{n} \sum_{i=1}^{n_{l}}\left(a_{t}^{i}-a_{t}^{j}\right)}{\left(1+l^{*}\right)^{2}-n_{l} \frac{l^{*}}{n}\left(1+l^{*}\right)} & b_{t}^{j}>0 \\ -A_{t}+a_{t}^{j}+\frac{l^{*}}{n} \sum_{i=1}^{n l} b_{t}^{i, l} & b_{t}^{j}<0\end{cases}
$$

where $l^{*}=(1-l) / l$ and $n^{l}$ is the number of lending banks.

From Figure 3 we see that lending constraints lead to an increase in the overnight interest rate on the last day of the maintenance period. In regime I, the equilibrium rate is given by

$$
i_{t}=E\left[i_{t+1}\right]+\left(i^{l}-E\left[i_{t+1}\right]\right) F\left(-A_{t}+\frac{l^{*}}{n} \sum_{i=1}^{n l} b_{t}^{i, l}\right)
$$

where $b_{t}^{i, l}>0$. Therefore, in both regimes lending constraints lead to higher interest rates.

The impact of lending constraints on the interbank market if markets are segmented is simulated in Section 8.2.

\section{How does frontloading work?}

As shown in the previous section, market frictions such as aggregate shocks or lending constraints increase interbank market rates. A central bank may wish to intervene through liquidity injections in order to steer the overnight interest rate towards the policy rate. The frontloading policy implemented by the ECB during the market turmoil in 2007/2008 consists of providing the additionally required liquidity in the early stage of the maintenance period and to adjust for liquidity imbalances at the end of the maintenance period. In this section we sketch how liquidity frontloading works. Our results rely on the regime-switching model and on the model for the fine-tuning operation on the last day of the maintenance period.

For illustration purposes let us consider a simplified model where the regime switch takes place on the last day of the maintenance period. Furthermore, assume that the liquidity imbalance $m=S+D_{T}-A_{T}$ on the last day $T$ is perfectly projected in regime I. We compare two scenarios:

Frontloading projected liquidity imbalances We assume that the central bank homogeneously frontloads the liquidity $m>0$ to each bank in regime I, i.e. due to the frontloading $m$ the current account of bank $j$ and the aggregate current account change to

$$
a_{t}^{j}+m
$$

\footnotetext{
${ }^{10}$ See Appendix Section C for the detailed derivation.
} 
and

$$
A_{t}^{j}+m
$$

respectively. If we apply the formula for interbank market trading in regime I (see equation (12)) we get

$$
b_{t}^{j}=-A_{t}-m+a_{t}^{j}+m=-A_{t}+a_{t} .
$$

i.e. interbank market trading remains unaffected by frontloading liquidity as the deviations of banks' current accounts $a_{t}^{j}+m$ to the aggregate current account $A_{t}+m$ do not change.

However, frontloading decreases equilibrium interest rates in regime I (cf. equation(18)) down to

$$
i_{t}=E\left[i_{t+1}\right]+\left(i^{l}-E\left[i_{t+1}\right]\right) F\left(-A_{t}-m\right) .
$$

Furthermore, the average deficiency $D_{T}$ is reduced as in interbank market equilibrium the individual deficiency is approximately reduced by (cf equation (1))

$$
\begin{aligned}
d_{t+1}^{j} & =\max \left(d_{t}^{j}-\max \left(a_{t+1}^{j}+m-b_{t}^{j}, 0\right), 0\right)=\max \left(d_{t}^{j}-\max \left(A_{t}+m+\lambda_{t}^{j}, 0\right), 0\right) \\
& \approx d_{t}^{j}-A_{t}-m-\lambda_{t}^{j} .
\end{aligned}
$$

Consequently, on the last day of the maintenance period, the required amount of the fine-tuning operation leading to interbank market rates equal the MBR is zero, i.e.

$$
f=2\left(S+D_{T}-m-A_{T}\right)=2\left(S+D_{T}-\left(S+D_{T}-A_{T}\right)-A_{T}\right)=0 .
$$

No frontloading In an alternative scenario, assume that the central bank does not frontload liquidity in regime I, i.e. $\mathrm{m}=0$. The amount of trading

$$
b_{t}^{j}=-A_{t}+a_{t}
$$

is the same as in the front-loading case (compare to equation 48) as in regime I the level of trading does not depend on the absolute level of the banks' current accounts $a_{t}^{j}$ but their deviation from the aggregate current account $A_{t}$. This observation that front-loading has no impact on the amount of interbank market trading in the early stage of the maintenance period (regime I) reflects our insight that in regime I banks only manage past liquidity shocks and not their deficiencies.

On the other hand, interest rates depend on the absolute level of the aggregate current account $A_{t}$ (see equation (12)) and therefore are higher in the no-frontloading case. Hence, we conclude that frontloading taking place in regime I has an impact on the interest rate level but not on the trading volume. 
Furthermore, in case of no-frontloading the final fine-tuning operation is given by

$$
f=2\left(S+D_{T}-A_{T}\right)=2 m
$$

i.e. it requires twice as much funds to reach the $\mathrm{MBR}$ on $t=T$ as compared to the frontloading scenario.

Policy implications We can draw two conclusion from this simple example.

First, the frontloading policy of the ECB leads to a decrease, and stabilizing effect, in the overnight interest rate. In the pre-crisis period in regime I the interbank market rate is given by equation (18), i.e. by

$$
i_{t}^{\text {pre-crisis }}=E\left[i_{t+1}\right]+\left(i^{l}-E\left[i_{t+1}\right]\right) F\left(-A_{t}\right) .
$$

As shown in section 6 in turmoil periods this relation changes to

$$
i_{t}^{\text {crisis-liquidityshock }}=E\left[i_{t+1}\right]+\left(i^{l}-E\left[i_{t+1}\right]\right) F\left(-A_{1}+\mu \cdot(t-1)\right)
$$

in case of an aggregate liquidity shocks $\mu$ and to

$$
i_{t}^{\text {crisis-lendingconstraints }}=E\left[i_{t+1}\right]+\left(i^{l}-E\left[i_{t+1}\right]\right) F\left(-A_{t}+\frac{l^{*}}{n} \sum_{i=1}^{n l} b_{t}^{i, l}\right)
$$

in case of lending constraints $l$. In both cases interest rates increase due to the corresponding market imperfection. If there is no further provision of liquidity in regime I, i.e. no frontloading, to compensate for the aggregate shock $\mu \cdot(t-1)$ and increased borrowing demand $\frac{l^{*}}{n} \sum_{i=1}^{n l} b_{t}^{i, l}$ as expressed in equation (55) and equation (56), respectively, interest rates go up in regime I. Furthermore, the formulas for interest rates in regime I also show that besides aggregate liquidity entering the term $F($.$) , the interest rate is also affected by expected future rates E\left[i_{t+1}\right]$. Therefore, a stabilizing policy has to consider both interest rate expectations and the current liquidity situation. The combined strategy of frontloading liquidity in regime I and eliminating remaining liquidity imbalances on $t=T$ via a fine-tuning operation, such that $i_{T}=M B R$, seems to be a suitable strategy to stabilize both effects.

Second, controlling expected rates is more efficient with frontloading. As shown in the simplified example above, frontloading excess reserves is more efficient in a "quantity" sense (e.g. smaller-scale central bank intervention) than providing excess reserves solely on the last day of the maintenance period. Moreover, this efficiency argument suggests the implementation of FTOs not only on $t=T$ but also during the maintenance period (as increasingly observed 
in the crisis period). ${ }^{11}$ In practice, implementing frontloading along the lines suggested by the simplified theoretical model is difficult because the central bank would have to forecast/know the future liquidity imbalance $m$ on the last day of the maintenance period. With increasing information in the course of the maintenance period forecasting errors about the imbalance on the lsat day will be reduced. The efficiency argument advises to correct forecasting errors in regime I (through fine-tuning operations or regular operations) when banks do not manage their deficiencies and only correct for liquidity shocks.

The arguments developed above are based on a very simplified model. Thus, in the next sections we consider a more realistic set-up which can be studied using a simulation framework.

\section{Calibration of the Model Parameters}

For simulating the model discussed in the previous section we estimate the following parameters for the pre-crisis and the crisis period:

- the average point of time at which banks switch from backward-looking liquidity shock correction to forward-looking reserve management

- the distribution parameters for the idiosyncratic liquidity shock $\lambda_{t}^{j} \sim N(\mu, \sigma)$

- the parameter for banks' lending constraint $l$

- the degree of market segmentation $s$.

The calibration is done in two main steps. ${ }^{12}$ Firstly, in Section 8.1, we estimate the timing of the regime-switch using survival analysis. For this purpose we use data on the daily end-of-day current accounts cuac $c_{t}^{j}$, minimum reserve requirements $m r r_{t}^{j}$ and net recourse to the standing facilities netr $r_{t}^{j}$ of $j=1, \ldots, 82$ euro area banks, covering the period between March 2003 and July $2007 .{ }^{13}$

Secondly, with the timing of the regime-switch fixed, we simulate the model with different combinations of parameters and market distortions, in order to find a combination of parameter values that minimises the Euclidian distance between simulated and realised values of EONIA

\footnotetext{
${ }^{11}$ We thank an anonymous referee for pointing out the importance of FTOs during the maintenance period during the crisis period.

${ }^{12}$ The models discussed in the previous section rely on the assumption that the distribution of the liquidity shocks $F$ is homogeneous across banks. To guarantee this assumption in the presence of differently sized banks, we consider liquidity shocks normalized by the adjusted minimum reserve requirement $R_{t}^{j}=\frac{M R R_{t}^{j}}{100}$. This normalization is discussed in greater detail in the appendix.

${ }^{13}$ From the original sample of 95 banks we excluded subsidiaries of international investment banks which show a pattern of liquidity management which is not comparable to the management of the majority of banks in the sample.
} 
and aggregate liquidity figures (See Section 8.2). The simulations are carried out along the lines suggested in [GQM08] and are conditional on aggregate liquidity data observed between March 2004 and September 2008.

The plausibility of the calibrated variance of the (idiosyncratic) liquidity shock distribution is cross-checked with an estimate of the volatility parameter using GMM methods applied to the panel bank data (see Appendix D).

\subsection{Timing of the regime-switch}

In section 4 we mentioned that the regime switch should depend on the (magnitude of the) probability of getting locked-in $P\left(d_{t}^{j}+b_{t}^{j}-a_{t}^{j}<\lambda_{t}^{j}\right)$. When this probability is below a certain threshold, $\epsilon$, each bank is assumed to manage only overdrafts, i.e. to keep, $b_{t}+a_{t}>0$, but not the deficiency, i.e. banks are in regime I. When the threshold is reached, the regime is switched and banks start to manage both deficiency and overdrafts.

While we cannot observe $a_{t}^{j}, b_{t}^{j}$ and $\lambda_{t}^{j}$ directly, the panel data set contains the end-of-day current account cuac $_{t}^{j}$ for bank $j$ given by

$$
\operatorname{cuac}_{t}^{j}=a_{t}^{j}-b_{t}^{j}+\lambda_{t}^{j} .
$$

Using this information we can infer the probability of the bank getting locked-in by

$$
P\left(d_{t}^{j}+b_{t}^{j}-a_{t}^{j}<\lambda_{t}^{j}\right)=P\left(d_{t}^{j}<\operatorname{cuac}_{t}^{j}\right)=1-P\left(\operatorname{cuac}_{t}^{j}<d_{t}^{j}\right)
$$

Hence, for the estimation of the probability of a bank getting locked-in we do not need to measure (unobservable) liquidity shocks or interbank market trading; in fact, all that is needed are the observable end-of-day current account $c u a c_{t}^{j}$ and deficiency $d_{t}^{j}$, which is included in our data set. The deficiency $d_{t}^{j}$ is calculated by

$$
d_{t}^{j}=\max \left(d_{t-1}^{j}-\max \left(\operatorname{cuac}_{t}^{j}-\text { netr }_{t}^{j}, 0\right), 0\right)
$$

where the net recourses to the standing facilities netr $r_{t}^{j}$ are taken into account. $d_{0}^{j}=n \cdot m r r_{t}^{j}$

with $n$ equal to the number of days in the maintenance period and $m r r_{t}^{j}$ is the minimum reserve requirement in this period.

Accordingly, the probability of an overdraft is given by

$$
P\left(\lambda_{t}^{j}<b_{t}^{j}-a_{t}^{j}\right)=P\left(c u a c_{t}^{j}<0\right)
$$

We approximate the cumulative distribution function $P\left(\right.$ cuac $\left._{t}^{j}<X\right)$ by an empirical cumulative distribution function $\hat{P}(X) \approx P\left(\operatorname{cuac}_{t}^{j}<X\right)$ for each bank $i$ with

$$
\hat{P}(X)=\frac{\sum_{t=1}^{T} I\left(\text { cuac }_{t}^{j}<X\right)}{T}
$$


where $I($.$) is an indicator function equaling one if the stated condition is fulfilled, otherwise$ being zero. The summary statistics of the distribution of the observed current accounts $c u a c_{t}^{j}$ is given in Table 1.

For the estimation of the timing of the regime switch we apply survival analysis. We calculate the probability of a bank getting locked-in $\hat{P}\left(d_{t}^{j}\right)$ and compare it to a threshold $\epsilon$. For different choices of the threshold $\epsilon$ we estimate the survival function

$$
S(\epsilon)_{t}=\operatorname{Prob}\left[\left(1-\hat{P}\left(d_{t}^{j}\right)\right)<\epsilon\right]
$$

where $S(\epsilon)_{t}$ is the probability that at time $t$ the probability of getting locked-in is below a threshold $\epsilon$, i.e. that the regime switch has not occurred up to time $t$. Time is measured in days until the end of the maintenance period. Depending on the magnitude of the deficiency and the current account distribution some banks may, and others may not, switch regime by the end of the maintenance period. Therefore, we apply the Kaplan-Meier estimator for nonparametric estimation of $S(t)$ in a right-censored problem.

Table 2 and Figure 10 plot the survival function for large, medium and small sized banks if (a) $\epsilon=0$ and $(\mathrm{b}) \epsilon=\hat{P}(0)$; i.e. banks start to manage their deficiency either when the probability of getting locked-in is (a) greater than zero or (b) greater than the probability of an overdraft. Table 2 compares the values of the survival function for the last week of the maintenance period, while Figure 10 plots the survival function over the entire maintenance period.

First of all, we observe that for different bank sizes the survival function has different values (see Figure 10). The log-rank test rejects the null hypothesis of equality of the survival functions of large, medium and small sized banks at the $1 \%$ significance level (this result is omitted in Table 2).

Figure 10 shows at which point of time the survival function deviates from 1 . If $\epsilon=0$ the first trading day when the survival function is below 1 is on the last Thursday, second last Monday and second last Thursday in the maintenance period for large, medium and small sized banks, respectively. If $\epsilon$ is set equal to the probability of an overdraft, the survival function starts to decline around one week before the end of the maintenance period for small and medium sized banks and approximately on the last Friday for large sized banks. In Table 2 we observe that on the last Friday of the MP around $60 \%$ of all banks are not locked in. Furthermore, we note that the last weekend in the maintenance period causes a jump in the regime switch probability. The deficiency on Monday, after a weekend, is calculated as

$$
d_{\text {Monday }}^{j}=\max \left(d_{\text {Friday }}^{j}-3 \max \left(a_{\text {Friday }}^{j}+\lambda_{\text {Friday }}^{j}-b_{\text {Friday }}^{j}, 0\right), 0\right)
$$


which implies that the deficiency jumps from Friday to Monday by $3 \max \left(a_{\text {Friday }}^{j}+\lambda_{\text {Friday }}^{j}-\right.$ $\left.b_{\text {Friday }}^{j}, 0\right)$. This weekend effect is especially relevant for the last weekend in the maintenance period: while on the last Friday in the maintenance period approximately $60 \%$ of all banks are not locked-in, on Monday, after the weekend, only $9 \%$ of all banks are not locked-in. This observation is in line with [GQM08] who show that the distribution of the EONIA on the last two to three days of the maintenance period differs from the distribution on the previous days of the maintenance period. For our simulation study we assume that the regime switch is on the last Monday of the MP for all banks.

\subsection{Simulations}

With the timing of the regime switch fixed around the last weekend in the reserve maintenance period, we systematically simulate different combinations of parameter values and scenarios of market frictions (lending constraints, market segmentation, aggregate liquidity shocks) in order to choose which one reproduces most closely, in simulations, the main empirical features and stylized facts of the euro overnight interbank market during the August 2007 - September 2008 period. The simulation design is similar to the one suggested by [GQM08]. ${ }^{14}$

We simulate the last 8 days of the maintenance period from Tuesday to Tuesday. This period covers 6 trading days and one weekend. For consideration of the latter we apply equation (62) for updating the deficiency on Monday. In this period the outstanding refinancing (MROs and LTROs) provided to the banks by the ECB is constant and, thus, the EONIA can be assumed to be determined only by banks' liquidity management according to the adapted model discussed in the first part of this paper. We apply the regime switching model described in Section 3 and Section 4 and assume that on the first 4 trading days (Tuesday to Friday) banks apply the backward-looking error (liquidity shock) correction model, while on the last two days (Monday and Tuesday) the supply of funds in the interbank market is determined by the forward-looking model $^{15}$.

We simulate 1000 maintenance periods. For the pre-crisis simulation we apply equation (12) for regime I. For regime II the integrals in equation (22) and equation (23) are approximated by Monte Carlo simulation with 500 simulation paths. A description of the algorithm applied for solving the stochastic dynamic programming problem at $t<T$ in regime 2 is described in

\footnotetext{
${ }^{14}$ However [GQM08] simulate only two days of active trading and neglect the last weekend effect.

${ }^{15}$ Note that this setting reflects our calibration findings based on our individual current account data set which only covers the pre-crisis period. In turmoil periods the average point of time for banks' regime-switch may take place earlier due to the frontloading of liquidity and banks' willingness to bearing the cost of early locking-in. In the aggregate data shown in Figure $8 \mathrm{a}$ and $8 \mathrm{~b}$ we observe increased usage of the deposit facility in the crisis period indicating higher locking-in in turmoil periods. A deeper investigation of banks' regime-switching behaviour in turmoil times is left to future research due to lack of data.
} 
the Appendix in Section F. To capture the effects of lending constraints, market segmentation and aggregate liquidity shocks in turmoil periods we apply the model modifications discussed in Section 6 .

We consider $n=60$ banks and initialize the state space by setting the banks' deficiencies $d_{T-7}^{j}$, current accounts $a_{T-7}^{j}$ and the targeted reserve surplus equal to empirically observed averages $D_{T-7}, A_{T-7}$ and $S$. We observe an average (adjusted) deficiency of $D_{T-7}=784$ $\left(D_{T-7}=723.5\right)$, an average current account of $A_{T-7}=101\left(A_{T-7}=96.5\right)$ and an accumulated reserve surplus of $S=14.5$ ( $S=12.0$ ) in the pre-crisis (crisis) period (quantities are given as multiples of the average minimum reserve requirement times 100).

By starting the simulations conditional on the aggregate deficiency $D_{T-7}$ and the average current account $A_{T-7}$, the central bank's liquidity frontloading is implicitly taken into account in the simulation. The higher the fraction $\frac{D_{T-7}}{A_{T-7}}$, the lower the amount of frontloading. Hence, in the pre-crisis period frontloading is given by $\frac{784}{101}=7.76$ and in the crisis period by $\frac{723.5}{96.5}=7.50$. The interest rate corridor of the standing facilities is set at $i^{d}=3 \%$ and $i^{l}=5 \%$, the level of ECB policy rates during most of the sample period. The amount of the fine-tuning operation $f$ on the last day $t=T$ is endogenously determined according to, $f=S-D_{T}+A_{T}$ (see Figure $9)$.

We carry out simulations assuming different parameter values for the liquidity shock distribution $\lambda_{t}^{j} \sim N(\mu, \sigma)$, the lending constraint $l$ and the degree of market segmentation $s$. For both the crisis and pre-crisis period we consider three assumptions for the liquidity shock volatility $\sigma \in\{30,45,55\}$. This range covers the empirically estimated individual liquidity shock volatility (see Section D). Liquidity shocks are generated such that $\sum_{j=1}^{n} \lambda_{t}^{j}=n \mu$, i.e. in case of no aggregate shocks, $\mu=0$, the impact of liquidity shocks on the aggregate current account $A_{T}$ is zero. For details on the generation of the liquidity shocks see [GQM08]. Furthermore, we test two scenarios of different degree of market segmentation $s$. We assume one scenario without frictions, i.e. $s=60$, and a scenario with market segmentation with 3 segments consisting of $s=60 / 3=20$ banks.

For each scenario we calculate the per capita deficiencies $D_{t}$, average end-of-day current accounts $A_{t}$ (excluding FTOs and after trading and receiving shocks) and the average amount of trading $|B|_{t}=\sum_{j=1}^{n}\left|b_{t}\right|$ as multiples of the minimum reserve requirement times 100 on the last 5 trading days of the maintenance period. Additionally we report the EONIA spread (EONIA - MBR) on each simulated trading day. For the last day of the maintenance period we report the lending/borrowing amount of the fine-tuning operation and if $l<1$ also the bid-ask spread 
spread $_{T}$.

For the determination of the EONIA on each simulated trading day we approximate the official calculation method by defining the simulated EONIA as the quantity-weighted average interest rate for all lending banks where the rate is weighted by the bank's amount of lending. As the EONIA is determined based on the reporting of unsecured lending transactions in the interbank market of banks with the highest business volumes in the Euro money markets, we focus on simulating the liquidity management of large size banks. The following scenarios are considered:

- Pre-crisis scenarios with and without market segmentation: $D_{T-7}=780, A_{T-7}=101$, $S=14.5$

- Crisis scenarios with and without market segmentation and increased frontloading: $D_{T-7}=$ 723.5, $A_{T-7}=96.5, S=12$.

- Crisis scenarios with and without market segmentation and negative aggregate shocks: $D_{T-7}=723.5, A_{T-7}=96.5, S=12$ analyzing the impact of an aggregate shock of $\mu=-0.5$.

- Crisis scenarios with and without market segmentation and lending constraints. $D_{T-7}=$ 723.5, $A_{T-7}=96.5, S=12$ ) and with lending constraints $l=0.97$ and 0.99 , respectively.

Table 3 reports the Euclidian distance between simulated and observed average EONIA spread, variation in the EONIA spread across maintenance-periods, shape of EONIA spread, and liquidity characteristics. For calibration we select the combination of parameters that minimises the Euclidian distance between simulated and observed values; the observed and simulated averages for the EONIA spread are illustrated in Figure 11.

The combination of parameters that minimises the choice criteria before the crisis is, $s=60$ (no market segmentation), $\sigma=45$, and no lending constraint $l=1$.

Therefore the simulations suggest that market segmentation and lending constraints were not a significant feature of the Euro inter-bank overnight market before the crisis. Moreover, the smooth declining path of EONIA observed towards the end of the reserve maintenance period, which documents a small deviation from the strong martingale hypothesis, can be explained in our model by the liquidity management regime-switch that occurs just after the last weekend in the period; it is only from that day onwards that banks start focusing on end-of-period conditions managing their reserve deficiency in a forward-looking manner. 
The combination of parameters that minimises the choice criterion EONIA spread during the crisis is, $s=20$ (market segmentation), $\sigma=45$, and lending constraint $l=0.99$, which gives (marginally) better results than those obtained with $l=0.97 .{ }^{16}$ However, given that the latter parameter gives lower Euclidian distances for the liquidity criterion, we chose $l=0.97$ for the calibration exercise.

It is interesting to note that simulations with the aggregate shock alone generate the third lowest criterion for the EONIA spread and the lowest criterion for the liquidity characteristics, with unchanged idiosyncratic volatility parameter from pre-crisis level $(\sigma=45)$. However, the aggregate liquidity shock alone does not allow reproducing in simulations the shape of the EONIA spread during the crisis; it is market segmentation that generates in most simulations a U-shape pattern for the EONIA spread.

Overall, the simulations suggest that a combination of market segmentation and lending constraints is needed in order for the model to replicate the observed patterns of trading in the interbank market during the crisis. The simulations suggest, somewhat surprisingly, that neither aggregate liquidity shocks nor increasing volatility of idiosyncratic liquidity shocks were the main features of the impact of the financial market crisis in the interbank overnight market as they under-perform compared to a combination of market segmentation and liquidity constraints in reproducing the shape and level of the EONIA spread during the crisis. Of course, in practice, it might be the case that all factors were present during the crisis.

\section{Policy evaluation}

In this section we evaluate different policies of providing and absorbing liquidity in the crisis period. For this purpose we simulate the last 8 days of 1000 maintenance periods conditional on different values for aggregate deficiency and current account combinations at the simulation starting point. Based on the results presented in the previous section, we calibrate liquidity shocks to be distributed according to $\lambda_{t}^{j} \sim N(0,45)$. Furthermore, we calibrate market segmentation at $s=20$ (3 banking groups) and lending constraints of $l=0.97$. Based on the empirical evidence banks' targeted accumulated excess reserves are set at $S=12$. The marginal lending rate (deposit rate) is assumed to be $i^{l}=5 \%\left(i^{d}=3 \%\right)$.

For policy evaluation we compare two different degrees of frontloading and two different systems of providing or absorbing liquidity on the last day of the maintenance period:

\footnotetext{
${ }^{16}$ The plausibility of the calibrated variance of the (idiosyncratic) liquidity shock distribution is cross-checked with an estimate of the volatility parameter using GMM methods applied to the panel bank data (see Appendix D)
} 
- We measure the degree of frontloading by the ratio of aggregate deficiency and aggregate current account observed after the last MRO in the maintenance period and implement different frontloading policies by starting the simulations conditional on different ratios of aggregate deficiency $D_{T-7}$ and current account $A_{T-7}$. We compare the aggregate frontloading policy observed in the crisis period $\left(A_{T-7}=96.5 / D_{T-7}=723.5\right)$, with a scenario generated by approximating $D_{T-7}$ and $A_{T-7}$ without frontloading. For generating the latter scenario we run the shock correction model for the first 15 trading days of the maintenance period (including weekends) with lending constraints $l=0.97$ and market segmentation $s=20$ assuming no change of medium or long term refinancing via MRO or LTRO. As a result we obtain the ratio $A_{T-7}=96.5 / D_{T-7}=807$ for the case of no frontloading in the crisis period.

- We compare the ECB's fine-tuning operation policy on the last day of the maintenance period with the reserve band system of the Bank of England discussed in Appendix E. For the simulation the reserve band is set to $r b=28 .{ }^{17}$ The volume of the fine-tuning operation $f$ on the last day $t=T$ is endogenously determined according to the estimated ECB rule (illustrated in Figure 9), i.e. $f \approx S-D_{T}+A_{T}$.

For each policy we calculate different criteria capturing the effectiveness and the efficiency of the different policies. The former measures how the policy performs in keeping EONIA close to the policy target (eg EONIA spread close to zero); the latter compare the policies by their degree of interference with market functioning.

- EONIA predictability: we calculate the EONIA spread over the MBR on the last day of the maintenance period and the time-series volatility of the EONIA over the last 5 trading days. A policy is considered to be more effective, if it leads to lower EONIA spread on the last day of the maintenance period, and lower EONIA standard deviation.

- Transaction costs: due to lending constraints banks trade at a bid-ask spread on the interbank market. The bid-ask spread is calculated according to equation (44). A policy is considered to be more effective, if it leads to a narrower bid-ask spread.

- Interbank market activity measured in average (absolute) trading volume during the last two days of the maintenance period. We only consider the trading volume in regime II as in regime I the average trading volume is independent of the applied policy as banks

\footnotetext{
${ }^{17} r b=28=0.01 \times M R R \times$ days in the $R M P$.
} 
only correct for liquidity shocks and do not manage reserves. A policy is considered to be more efficient, if it leads to larger trading volumes.

- Liquidity Provision: by calculating the liquidity provided/absorbed on the last day of the maintenance period by the central bank and by comparing the total amount of liquidity provision including the amount of frontloaded liquidity we focus on the supply side of each policy. A policy is considered to be more efficient, if it requires less funds to be provided to the market by the central bank.

Figure 12 plots the EONIA over the last 5 trading days for the four evaluated policies. Table 6 compares the benchmark policy defined to be frontloading plus fine-tuning operation on $\mathrm{t}=\mathrm{T}$ as observed in the crisis period to policies with and without frontloading combined with reserve band system or fine-tuning operation on the last day of the maintenance period. For each quality criterion the average value over all simulated maintenance periods and the difference to the benchmark policy is given. ${ }^{18}$

First we analyze the case when there is no frontloading in the crisis period scenario. In this case the entire liquidity (approx. $61 \%$ of the MRR) is provided on the last day of the maintenance period (see Table 6), where there is no significant difference between fine-tuning operation and reserve band system (not reported in the table). The total liquidity provided exceeds the "observed frontloading" case by about 15-16\% points. Furthermore, if there was no frontloading in the crisis period the EONIA would have been approximately 23 bps higher on the last day of the maintenance period (in case of a fine-tuning operation on the last day of the maintenance period). The impact of no frontloading would have been even higher if the (symmetric) reserve band system was adopted instead of the fine-tuning operation. In this case the EONIA would have been approximately 38 bps higher. Moreover, no frontloading leads to higher EONIA time-series standard deviation and higher bid-ask spread which is approximately 0.7-1.2 basis points higher compared to the observed frontloading scenarios (see Table 6 and Figure 12). Interbank market trading activity measured by average trading volume is also lower if no frontloading is carried out. Depending on whether a FTO or the reserve band system is adopted on the last day of the maintenance period, the interbank market trading is 4-9 percentage points lower than in the observed frontloading scenario. Hence, we conclude that irrespective of whether a fine-tuning operation is conducted or a reserve band system is in place, on the last day of the maintenance period, if there is no liquidity provided in the early stage of

\footnotetext{
${ }^{18}$ We refer to quality of policy rather than welfare analysis because the latter is associated with general equilibrium, whereas the scope of ours is partial (dis)equilibrium analysis.
} 
the maintenance period and if markets are not frictionless, liquidity provision only on the last day of the maintenance period leads to higher total liquidity provision by the central bank. This confirms the results of Section 7 for markets with frictions. Furthermore, and more importantly, even with increased liquidity provision in case of no frontloading, the EONIA, transaction costs and the interbank market activity cannot be steered as close to the targets as it is in the case of frontloading. For instance, considering the EONIA spread, even with liquidity provision of $61 \%$ of the MRR the EONIA spread cannot be steered towards zero due to market segmentation and lending constraints.

Comparing the fine-tuning operation and the reserve band system in case of frontloading as observed, we note that the reserve band produces slightly better results for all quality criteria (statistically significant difference). The reserve band system absorbs slightly more liquidity on the last day of the maintenance period ( $-3.3 \%$ of the MRR compared to $-3.0 \%$ in case of a FTO), leading to a more efficient use of liquidity, higher average interbank market trading, lower transaction costs and lower EONIA standard deviation. Nevertheless, both the fine-tuning operation and the reserve band lead to EONIA spreads which are not statistically different from zero on the last day of the maintenance period.

\section{Conclusions}

In the first part of this paper we extend the [QM06] model of the euro overnight interbank market in three directions. First, we incorporate the microstructure of the European interbank market to capture the level and the shape of the Euro interbank market rate. Second, we propose a regime switching model with endogenous regime switch which allows to simulate the entire maintenance period without facing the problem of curse of dimensionality. Moreover, the model provides theoretical insights into the trading behavior of banks in the early stage of the maintenance period. Thirdly, we propose a model for an interbank market which is distorted by credit rationing and show that lending constraints of banks lead to a bid-ask spread on the interbank market.

In the second part we use our model to study the impact of the market turmoil on the euro overnight interbank market by allowing for market segmentation, lenders' credit lines (credit rationing), and aggregate liquidity shocks. The main conclusion of the calibration and subsequent simulations is that increasing liquidity volatility (idiosyncratic or aggregate) is neither necessary nor sufficient to generate the kind of market developments that were observed during the crisis. In fact, for the model to replicate the kind of interbank trading patterns observed 
during turmoil what is needed is a combination of (a) increasing market segmentation, which leads to U-shape pattern of the EONIA and increased EONIA volatility; and (b) credit limits (rationing) which increase the level of the EONIA.

Using panel data of 82 Euro Area commercial banks covering the period March 2003 - July 2007 we find that (i) the average idiosyncratic liquidity shock volatility is around $45 \%$ of the minimum reserve requirement and that (ii) banks on average switch from a backward-looking liquidity shock correction regime to a forward-looking reserve management regime around the last weekend of the maintenance period. The latter result gives one explanation to the observation in [GQM08] that the EONIA distribution on the last three days is different from the EONIA distribution on previous days of the maintenance period.

Based on simulations of market segmentation and lending constraints, we evaluate ECB's liquidity frontloading policy and compare it with a reserve band system policy similar to the Bank of England's framework. The simulation results suggest that without frontloading the overnight interest rate would have been, on average, 23 basis points above the policy rate (target); with frontloading and a fine-tuning operation on the last day of the maintenance period, the overnight rate is, on average, on target. Therefore, our theoretical result that frontloading is an efficient tool to stabilize interest rates, is also confirmed for distorted markets. Comparing the finetuning policy of the ECB to a reserve band system similar to the framework of the Bank of England we find that while both are equally able to anchor the overnight rate on the last day of the maintenance period, the latter seems to improve over the former in terms of higher average interbank market trading, lower transaction costs and lower EONIA standard deviation.

There is an additional insight provided by our analysis. With frontloading the central bank fosters market activity and steers the overnight interest rate; however, the source of market distortions, captured in the model by market segmentation and lending constraints, are not addressed / impacted by the policy as they are kept as exogenous parameters in the simulations. This only can be justified if those distortions have their roots in solvency, rather than liquidity problems. To the extent that our model captures the main features of the impact of the financial market turmoil on the functioning of the interbank market, it highlights as well the limits to central bank policy focused on liquidity management. 


\section{References}

[BCMHW06] U. Bindseil, G. Camba-Mendez, A. Hiersch, and B. Weller. Excess reserves and the implementation of monetary policy of the ecb. Journal of Policy Modeling, 28:491-510, 2006 .

[ECB06] ECB. The eurosystem's experience with fine-tuning operations at the end of the reserve maintenance period. ECB Monthly Bulletin, November, pages 83-91, 2006.

[GQM08] V. Gaspar, G. Quirós, and H. Mendizábal. Interest rate dispersion and volatility in the market for daily funds. European Economic Review, 52(3):413-440, 2008.

[HM82] R. Hall and F. Mishkin. The sensitivity of consumption to transitory income: Estimates from panel data on households. Econometrica, 50(2):461-481, 1982.

[Mac05a] S. MacGorain. Achieving overnight rate stability in a reserves averaging framework. Conference paper, ECB Conference on Monetary Policy Implementation, 20-21 January 2005, 2005.

[Mac05b] S. MacGorain. Stabilising short-term interest rates. Bank of England Quarterly Bulletin, Winter, pages 462-470, 2005.

[Poo68] W. Poole. Commercial bank reserve management in a stochastic model: implications for monetary policy. Journal of Finance, 23(5):769-791, 1968.

[QM06] G. Quirós and H. Mendizábal. The daily market for funds in europe: what has changed with the emu? Journal of Money, Credit, and Banking, 38(1):91-118, 2006. 


\section{A Minimum reserve requirement adjustment}

To ensure the assumption that the distribution for the liquidity shocks $F$ is homogeneous across banks in the presence of different sized banks in our sample we consider liquidity shocks which are normalized by the adjusted minimum reserve requirement $R_{t}^{j}=\frac{M R R_{t}^{j}}{100}$, i.e. by

$$
\lambda_{t}^{j *}=\frac{\lambda_{t}^{j}}{R_{t}^{j}}
$$

We further assume ${ }^{19}$ that the shocks are distributed such that

$$
F\left(b_{t}^{j}-a_{t}^{j}\right)=F^{*}\left(\frac{b_{t}^{j}-a_{t}^{j}}{R_{t}^{j}}\right)
$$

where $\lambda_{t}^{* j} \sim F^{*}$ and $\lambda_{t}^{j} \sim F$. Then equation (10) becomes

$$
b_{t}^{j}=a_{t}^{j}+R_{t}^{j} F^{*-1}\left(\frac{i_{t}^{j}-E\left[i_{t+1}\right]}{i^{l}-E\left[i_{t+1}\right]}\right) .
$$

If the interbank market is cleared at the rate $i_{t}^{j}=i_{t}$, then

$$
0=\sum_{j=1}^{n} b_{t}^{j}=\sum_{j=1}^{n} a_{t}^{j}+\sum_{j=1}^{n} R_{t}^{j} F^{*-1}\left(\frac{i_{t}-E\left[i_{t+1}\right]}{i^{l}-E\left[i_{t+1}\right]}\right) .
$$

Consequently, the interbank trading as fraction of the minimum reserve requirement is given by

$$
b_{t}^{* j}=\frac{b_{t}^{j}}{R_{t}^{j}}=-A_{t}^{*}+\frac{a_{t}^{j}}{R_{t}^{j}}
$$

where

$$
A_{t}^{*}=\frac{\sum_{j=1}^{n} a_{t}^{j}}{\sum_{j=1}^{n} R_{t}^{j}} .
$$

\section{B Optimization problem for the fine-tuning operation}

If $f<0$, i.e. in case of a liquidity surplus, the $\operatorname{costs} c_{T}^{j}$ of a bank using the ECB's standing facilities at $t=T$ are given by borrowing at the marginal lending facility at the rate $i^{l}$ up to the targeted accumulated excess reserves $S$, i.e. if $a_{T}^{j}-d_{T}^{j}-b_{T}^{j}+\lambda_{T}^{j}<S$, or by lending at the

${ }^{19}$ For instance, if $F$ and $F^{*}$ are normal distributions, then

$$
F^{*}\left(\frac{b_{t}^{j}-a_{t}^{j}}{R_{t}^{j}}\right)=\Phi\left(\frac{\frac{b_{t}^{j}-a_{t}^{j}-\mu_{\lambda}}{R_{t}^{j}}}{\sigma_{\lambda} / R_{t}^{j}}\right)=\Phi\left(\frac{b_{t}^{j}-a_{t}^{j}-\mu_{\lambda}}{\sigma_{\lambda}}\right)=F\left(b_{t}^{j}-a_{t}^{j}\right)
$$


rate $i^{f}$ up to an amount of $-f$ and lending the remaining surplus at the rate $i^{d}$ such that the targeted accumulated excess reserves $S$ are met, i.e.

$$
\begin{aligned}
c_{T}^{j} & =i^{l}\left(d_{T}^{j}+b_{T}^{j}-a_{T}^{j}-\lambda_{T}^{j}\right) I\left(a_{T}^{j}-b_{T}^{j}+\lambda_{T}^{j}-d_{T}^{j}<S\right) \\
& -i^{f}\left(a_{T}^{j}-b_{T}^{j}+\lambda_{T}^{j}-d_{T}^{j}-S\right) I\left(S<a_{T}^{j}-b_{T}^{j}+\lambda_{T}^{j}-d_{T}^{j}<S-f\right) \\
& -\left(i^{d}\left(a_{T}^{j}-b_{T}^{j}+\lambda_{T}^{j}-S+f-d_{T}^{j}\right)-i^{f} f\right) I\left(S-f<a_{T}^{j}-b_{T}^{j}+\lambda_{T}^{j}-d_{T}^{j}\right)
\end{aligned}
$$

where $I(x)$ is an indicator function equalling 1 if the condition $x$ is true, and 0 otherwise. The expected profit is given by

$$
\begin{aligned}
& E\left[i_{T} b_{T}^{j}-c_{T}^{j}\right]=i_{T} b_{T}^{j}-i^{l} \int_{-\infty}^{M_{T}^{j}}\left(M_{T}^{j}-\lambda_{T}^{j}\right) f\left(\lambda_{T}\right) d \lambda_{T} \\
+ & \int_{M_{T}^{j}-f}^{\infty}\left(i^{d}\left(-M_{T}^{j}+\lambda_{T}^{j}+f\right)-i^{f} f\right) f\left(\lambda_{T}\right) d \lambda_{T}+\int_{M_{T}^{j}}^{M_{T}^{j}-f} i^{f}\left(-M_{T}^{j}+\lambda_{T}^{j}\right) f\left(\lambda_{T}\right) d \lambda_{T} \\
= & i_{T} b_{T}^{j}-i^{l}\left(M_{T}^{j}\right) F\left(M_{T}^{j}\right)+i^{l} \int_{-\infty}^{M_{T}^{j}} \lambda_{T}^{j} f\left(\lambda_{T}\right) d \lambda_{T} \\
+ & \left(i^{d}\left(-M_{T}^{j}+f\right)-i^{f} f\right)\left(1-F\left(M_{T}^{j}-f\right)\right)+i^{d} \int_{M_{T}^{j}-f}^{\infty} \lambda_{T}^{j} f\left(\lambda_{T}\right) d \lambda_{T} \\
+ & i^{f}\left(-M_{T}^{j}\right)\left(F\left(M_{T}^{j}-f\right)-F\left(M_{T}^{j}\right)\right)+i^{f} \int_{M_{T}^{j}}^{M_{T}^{j}-f} \lambda_{T}^{j} f\left(\lambda_{T}\right) d \lambda_{T}
\end{aligned}
$$

where $M_{T}^{j}=d_{T}-a_{T}^{j}+b_{T}^{j}+S$. The first order condition for an optimum is given by

$$
\begin{aligned}
\frac{\partial E\left[i_{T} b_{T}^{j}-c_{T}^{j}\right]}{\partial b_{T}^{j}} & =i_{T}^{j}-i^{d}-\left(i^{l}-i^{f}\right) F\left(d_{T}^{j}+b_{T}^{j}-a_{T}^{j}+S\right)-\left(i^{f}-i^{d}\right) F\left(d_{T}^{j}-f+b_{T}^{j}-a_{T}^{j}+S\right) \\
& =0
\end{aligned}
$$

In case of a liquidity deficit, i.e. if $f>0$, the $\operatorname{costs} c_{T}^{j}$ are calculated as the cost of borrowing, where up to the amount $f$ the rate $i^{f}$ applies and for the remaining amount of borrowing the rate $i^{l}$ has to be paid. The costs of lending are given by the rate $i^{d}$. Both borrowing and lending banks are assumed to target accumulated excess reserves of $S$. Then, the costs are given by

$$
\begin{aligned}
c_{T}^{j} & =\left(i^{l}\left(S+d_{T}^{j}+b_{T}^{j}-a_{T}^{j}-\lambda_{T}^{j}-f\right)+i^{f} f\right) I\left(a_{T}^{j}-b_{T}^{j}+\lambda_{T}^{j}-d_{T}^{j}<S-f\right) \\
& +i^{f}\left(S+d_{T}^{j}+b_{T}^{j}-a_{T}^{j}-\lambda_{T}^{j}\right) I\left(S-f<a_{T}^{j}-b_{T}^{j}+\lambda_{T}^{j}-d_{T}^{j}<S\right) \\
& -i^{d}\left(a_{T}^{j}-b_{T}^{j}+\lambda_{T}^{j}-d_{T}^{j}-S\right) I\left(S<a_{T}^{j}-b_{T}^{j}+\lambda_{T}^{j}-d_{T}^{j}\right) .
\end{aligned}
$$


The expected profit is given by

$$
\begin{aligned}
& E\left[i_{T} b_{T}^{j}-c_{T}^{j}\right]=i_{T} b_{T}^{j}-i^{f} \int_{M_{T}^{j}-f}^{M_{T}^{j}}\left(M_{T}^{j}-\lambda_{T}^{j}\right) f\left(\lambda_{T}\right) d \lambda_{T} \\
- & \int_{-\infty}^{M_{T}^{j}-f}\left(i^{l}\left(M_{T}^{j}-\lambda_{T}^{j}-f\right)+i^{f} f\right) f\left(\lambda_{T}\right) d \lambda_{T}+\int_{M_{T}^{j}}^{\infty} i^{d}\left(-M_{T}^{j}+\lambda_{T}^{j}\right) f\left(\lambda_{T}\right) d \lambda_{T} \\
= & i_{T} b_{T}^{j}-i^{f}\left(M_{T}^{j}\right)\left(F\left(M_{T}^{j}\right)-F\left(M_{T}^{j}-f\right)\right)+i^{f} \int_{T}^{M_{T}^{j}} \lambda_{T}^{j} f\left(\lambda_{T}\right) d \lambda_{T} \\
- & \left(i^{l}\left(M_{T}^{j}-f\right)-i^{f} f^{j}\right) F\left(M_{T}^{j}-f\right)+i^{l} \int_{-\infty}^{M_{T}^{j}-f} \lambda_{T}^{j} f\left(\lambda_{T}\right) d \lambda_{T} \\
+ & i^{d}\left(-M_{T}^{j}\right)\left(1-F\left(M_{T}^{j}\right)\right)+i^{d} \int_{M_{T}^{j}}^{\infty} \lambda_{T}^{j} f\left(\lambda_{T}\right) d \lambda_{T}
\end{aligned}
$$

The first order condition for an optimum is given by

$$
\begin{aligned}
\frac{\partial E\left[i_{T} b_{T}^{j}-c_{T}^{j}\right]}{\partial b_{T}^{j}} & =i_{T}^{j}-i^{d}-\left(i^{l}-i^{f}\right) F\left(d_{T}^{j}-f+b_{T}^{j}-a_{T}^{j}+S\right)-\left(i^{f}-i^{d}\right) F\left(d_{T}^{j}+b_{T}^{j}-a_{T}^{j}+S\right) \\
& =0
\end{aligned}
$$

\section{Inter-bank market trading in case of lending constraints}

In the shock correction model we assume that the interbank market trading is determined by

$$
b_{t}^{j}= \begin{cases}-b_{t}^{j-}+a_{t}^{j}+F^{-1}\left(\frac{i_{t}^{j}-E\left[i_{t+1}\right]}{i^{l}-E\left[i_{t+1}\right]}\right) & b_{t}^{j}>0 \\ a_{t}^{j}+F^{-1}\left(\frac{i_{t}^{j}-E\left[i_{t+1}\right]}{i^{l}-E\left[i_{t+1}\right]}\right) & b_{t}^{j}<0\end{cases}
$$

where $b_{t}^{j-}$ is the non-interbank-market lending.

By setting

$$
0=\sum_{j=1}^{n} b_{t}^{j}=A_{t}-\frac{\sum_{j=1}^{n_{l}} b_{t}^{j-}}{n}+F^{-1}\left(\frac{i_{t}-E\left[i_{t+1}\right]}{i^{l}-E\left[i_{t+1}\right]}\right) .
$$

we get

$$
\min \left(b_{t}^{j}, b_{t}^{j} l\right)= \begin{cases}-A_{t}+a_{t}^{j}+\frac{\sum_{j=1}^{n_{l}} b_{t}^{j-}}{n}-b_{t}^{j-} & b_{t}^{j}>0 \\ -A_{t}+a_{t}^{j}+\frac{\sum_{j=1}^{n_{l}} b_{t}^{j-}}{n} & b_{t}^{j}<0\end{cases}
$$

By further assuming that $b_{t}^{j-}=b_{t}^{j}(1-l)$ and solving the equation system we obtain

$$
b_{t}^{j}= \begin{cases}\frac{\left(-A_{t}+a_{t}^{j}\right)\left(1+l^{*}\right)+\frac{l^{*}}{n} \sum_{i=1}^{n}\left(a_{t}^{i}-a_{t}^{j}\right)}{\left(1+l^{*}\right)^{2}-n_{l} \frac{l^{*}}{n}\left(1+l^{*}\right)} & b_{t}^{j}>0 \\ -A_{t}+a_{t}^{j}+\frac{l^{*}}{n} \sum_{i=1}^{n l} b_{t}^{i, l} & b_{t}^{j}<0\end{cases}
$$




\section{Liquidity Shock Volatility}

For cross-checking the validity of the calibrated idiosyncratic shock volatility parameter we estimate the volatility parameter directly applying GMM to the panel bank data covering the period March 2003 - July 2007.

The $i$-th bank's observable current account cuac $_{t}^{i}$ after trading during regime I and receiving the liquidity shock is given by

$$
\operatorname{cuac}_{t}^{i}=a_{t}^{i}-b_{t}^{i}+\lambda_{t}^{i}
$$

Guided by the theory derived in Section 4 we model interbank market trading by

$$
b_{t}^{i}=-A_{t}+\beta a_{t}^{i}+\epsilon_{t} .
$$

This specification is motivated by the observation of banks targeting liquidity excess reserves, where $X=(\beta-1) a^{i}+\epsilon($ see Section 5$)$.

Then the current account is given by

$$
\operatorname{cuac}_{t}^{i}=(1-\beta) a_{t}^{i}+A_{t}+\lambda_{t}^{i}+\epsilon_{t}
$$

Inserting equation (70) into equation (69) and taking first differences leads to

$$
\begin{aligned}
\Delta \operatorname{cuac}_{t+1}^{i} & =A_{t+1}-A_{t}+\lambda_{t+1}^{i}-\lambda_{t}^{i}+(1-\beta)\left(a_{t+1}^{i}-a_{t}^{i}\right)+\Delta \epsilon_{t+1} \\
& =A_{t+1}-A_{t}+\lambda_{t+1}^{i}-\beta \lambda_{t}^{i}+\Delta \epsilon_{t+1}
\end{aligned}
$$

For model estimation we lean on the idea of covariance estimation for panel data proposed by [HM82]. We proceed in two steps: we first run OLS regressions on $\Delta c u a c_{t}^{j}$ to filter MRO and LTRO changes and to extract the corresponding residuals $\Delta \overline{c u a c}_{t}^{i}$ which we consider to contain the pure information about liquidity shocks $\lambda_{t}^{i}$. In a second step we estimate the covariance structure of the residuals $\Delta \overline{c u a c}_{t}^{i}$ with a parametric function of market segmentation $s$, the trading parameter $\beta$, and shock liquidity variance $\sigma_{\lambda}^{2}$.

Considering market segmentation as introduced by [GQM08] we get

$$
\Delta \operatorname{cuac}_{t+1}^{i}=\frac{\sum_{j=1}^{s} \lambda_{t}^{j}}{s}+\lambda_{t+1}^{i}-\beta \lambda_{t}^{i}+\Delta \epsilon_{t+1}
$$

where we used the fact that $a_{t+1}^{i}-a_{t}^{i}=\lambda_{t}^{i}$ and $A_{t}=\frac{\sum_{i=1}^{n} a_{t}^{i}}{n}$. We additionally consider banks to have different idiosyncratic shock volatilities. If shock volatility varies with bank size, we estimate $\sigma_{\lambda, \text { large }}, \sigma_{\lambda, \text { medium }}$ and $\sigma_{\lambda, \text { small }}$ for large, medium and small banks, respectively. By the law of total variance we get for the shock volatility

$$
\sigma_{\lambda}^{* 2}=\sigma_{\lambda, \text { large }}^{2} p_{\text {large }}+\sigma_{\lambda, \text { medium }}^{2} p_{\text {medium }}+\sigma_{\lambda, \text { small }}^{2} p_{\text {small }}
$$


where $p$. is the probability of occurrence. By assuming $\rho\left(\lambda_{t}^{j}, \lambda_{t}^{i}\right)=-\frac{1}{n-1}$ for $i \neq j, \operatorname{Var}\left(\epsilon_{t}^{i}\right)=\sigma_{\epsilon}^{2}$ the covariance structure $\Omega(\theta)$ with $\theta=\left(\sigma_{\lambda}, s, \beta\right)$ is specified as

$$
\begin{aligned}
\operatorname{Var}\left(\Delta \overline{\operatorname{cuac}}_{t+1}^{s}\right)= & \left(\frac{(s-1)}{s^{2}}-\frac{(s-1)(s-2)}{(n-1) s^{2}}\right) \sigma_{\lambda}^{* 2} \\
& -\frac{2(s-1)\left(\frac{1}{s}-\beta\right) \sigma_{\lambda, s} \sigma_{\lambda}^{*}}{(n-1) s}+\left(1+\left(\frac{1}{s}-\beta\right)^{2}\right) \sigma_{\lambda, s}^{2}+2 \sigma_{\epsilon}^{2} \\
\operatorname{Cov}\left(\Delta \overline{\operatorname{cuac}}_{t+1}^{j}, \Delta \overline{\operatorname{cuac}}_{t}^{j}\right)= & \left(\frac{1}{s}-\beta\right) \sigma_{\lambda, s}^{2}-\frac{1-\frac{1}{s}}{n-1} \sigma_{\lambda, s} \sigma_{\lambda}^{*}-\sigma_{\epsilon}^{2}
\end{aligned}
$$

where we assumed that $E\left[\epsilon_{t} \lambda_{t}\right]=0$

For filtering medium and long term effects we carry out an OLS regression to obtain the residuals $\overline{c u a c}_{t}^{i}$, i.e.

$$
\Delta \operatorname{cuac}_{t}^{i}=\alpha+\beta^{M R O} \Delta M R O_{t}+\beta^{L T R O} \Delta L T R O_{t}+\Delta \overline{c u a c}_{t}^{i} .
$$

The residuals $\Delta \overline{\mathrm{cuac}}_{t}^{i}$ of the time series regressions of each banks' current account changes are taken as input for the GMM estimation. Distribution moments, normality test and autocorrelation for the residuals $\Delta \overline{c u a c}_{t}^{i}$ are given in Table 4 .

In Table 5 we report the results of the GMM estimation of the model parameters. We find the idiosyncratic volatility to be around $42 \%$ for large banks, $38 \%$ for medium sized banks and $36 \%$ for small banks. The J-test indicates that the moment conditions specified above fit the data. Note that market segmentation has almost no impact on the estimation results. The main conclusion is that the calibrated volatility parameter, $\sigma=45$, is close to the value estimated for the large banks in the sample.

\section{E Reserve band system}

Within the reserve band system of the Bank of England, each bank is not required to meet the reserve requirements exactly but only within a band width of $\pm 1 \%$ of the MRR. If the reserves are within the band they are remunerated by the rate $i^{r}$ equaling the official interest rate. $[\mathrm{Mac} 05 \mathrm{~b}]$ and $[\mathrm{Mac} 05 \mathrm{a}]$ provide formulas for the optimal interbank market trading in the [QM06] model. We model a similar policy where we assume that the remuneration rate $i^{r}$ equals the rate $\frac{i^{l}+i^{d}}{2}$ and that the band width is equal to

$$
r b=0.01 \cdot M R R \cdot \text { days in maintenance period }
$$

i.e. equal to the accumulated maximum excess reserves remunerated at $i^{r}$. Then by adapting the formulas of [Mac05b] and [Mac05a] the interbank rate and trading on the last day of the 
maintenance period are determined by

$$
i_{T}^{j}=i^{d}+\frac{i^{l}-i^{d}}{2}\left(F\left(-r b+S+d_{T}^{j}+b_{T}^{j}-a_{T}^{j}\right)+F\left(r b+S+d_{T}^{j}+b_{T}^{j}-a_{T}^{j}\right)\right)
$$

if the deficiency of bank $j$ is outside the reserve band, and by

$$
i_{T}^{j}=i^{d}+\frac{i^{l}-i^{d}}{2}\left(F\left(b_{T}^{j}-a_{T}^{j}\right)+F\left(r b+S+d_{T}^{j}+b_{T}^{j}-a_{T}^{j}\right)\right)
$$

if the deficiency of bank $j$ is inside the reserve band (if the bank is inside the band it has to avoid an overdraft, i.e. it has to ensure that $a_{T}^{j}-b_{T}^{j}>0$ otherwise it has to borrow funds at the marginal lending facility). Figure 4 compares the demand curves of the fine-tuning operation and the reserve band system in an example. In this example, if a bank in a reserve band system

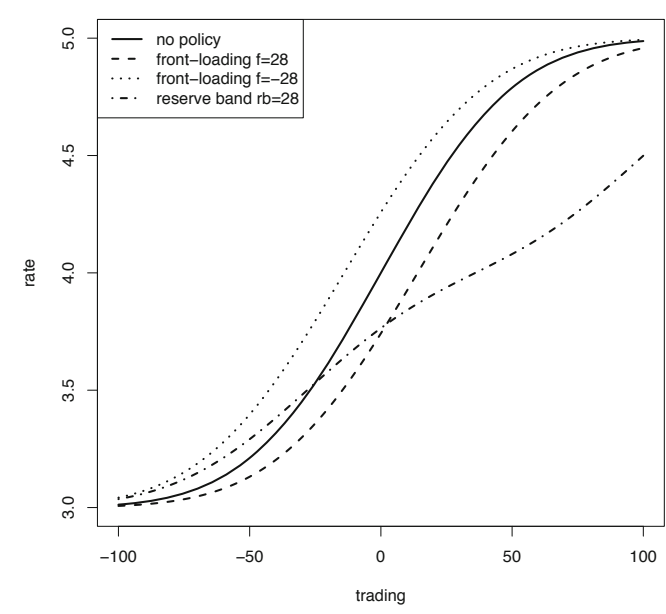

(a) $\left|D_{T}-A_{T}\right|<r b$

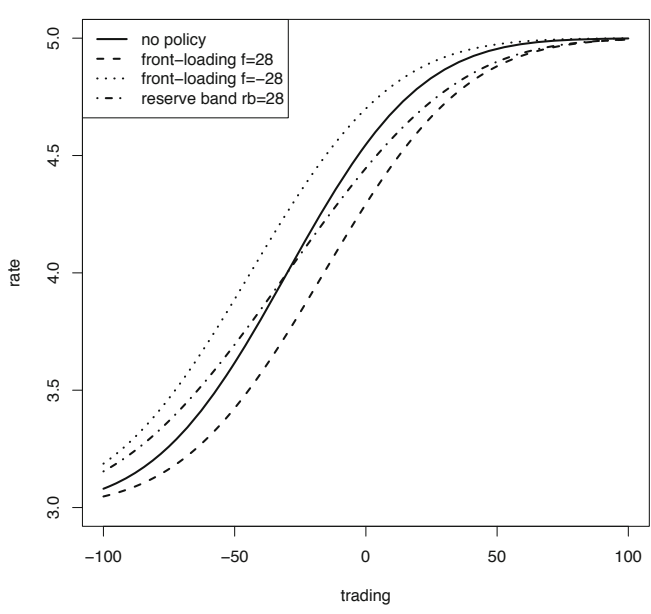

(b) $\left|D_{T}-A_{T}\right|>r b$

Figure 4: Demand curve for $i^{d}=3 \%, i^{l}=5 \%, D_{T}^{j}=100, A_{T}^{j}=100, f=0, S=0, \lambda_{T}^{j} \sim$ $N(0,40)$.

ends within the reserve band on the last day of the maintenance period, optimal lending is at a lower rate than in the fine-tuning operation system. For banks with deficiency outside the band, the individual lending and borrowing rates in a reserve band system are bounded from below and above by rates resulting from liquidity providing and liquidity absorbing fine-tuning operations, respectively, in a non-reserve band system.

\section{F Description of the Solution Algorithm}

On the pen-ultimate day $t=T-1$ of the maintenance period a system of equations of the form $i_{T-1}^{j}=f\left(b_{T-1}^{j}, d_{T-1}^{j}, a_{T-1}^{j}, E\left(i_{T}\right)\right)$ with $j=1, \ldots, n$ has to be solved to obtain the optimal 
trading $b_{T-1}^{j}$ for each bank such that the market is cleared. The calculation requires to calculate the expected value of the rate $i_{T}=f\left(b_{T-1}, b_{T}, a_{T-1}, d_{T-1}, \lambda_{T-1}\right)$ where $b_{T-1}, b_{T}, d_{T}$ and $a_{T}$ are $n$-dimensional vectors of trading, deficiency and current accounts at $T$ and $T-1$, respectively. For solving this problem we fix a rate $\hat{i}_{T}$ and determine the market clearing trading vector $\hat{b}_{T-1}$ for $i_{T}=\hat{i}_{T}$. Conditional on $\hat{b}_{T-1}$ we calculate the rate $i_{T}$. We repeat this procedure systematically until the difference $\hat{i}_{T}-i_{T}<t o l$ where $t o l$ is a tolerance level.

For the determination of market clearing trading $b_{t}$ we generate a grid where the entry $b(k, j)$ gives for bank $j=1, \ldots, n$ the optimal trading $b_{T-1}^{j}$ at the individual optimal rate $i_{k}=i_{T-1}^{j}$ with $i^{k}=\left\{i^{d}, i^{d}+\frac{i^{l}-i^{d}}{m-1}, \ldots, i^{l}-\frac{i^{l}-i^{d}}{m-1}, i^{l}\right\}$. The optimal trading is given by $b_{t}^{j}=b(k, j)$ with $k=\operatorname{argmin}_{h}\left|\sum_{j=1}^{n}(h, j)\right|$, i.e. where the market imbalance is lowest. Although this procedure is computational expensive it allows to consider trading constraints in the determination of the optimal market clearing market vector. An example for a trading restriction is the constraint that current accounts are not allowed to be negative, i.e. $a_{t}^{j}-b_{t}^{j}>0$. 


\section{G Figures and Tables}
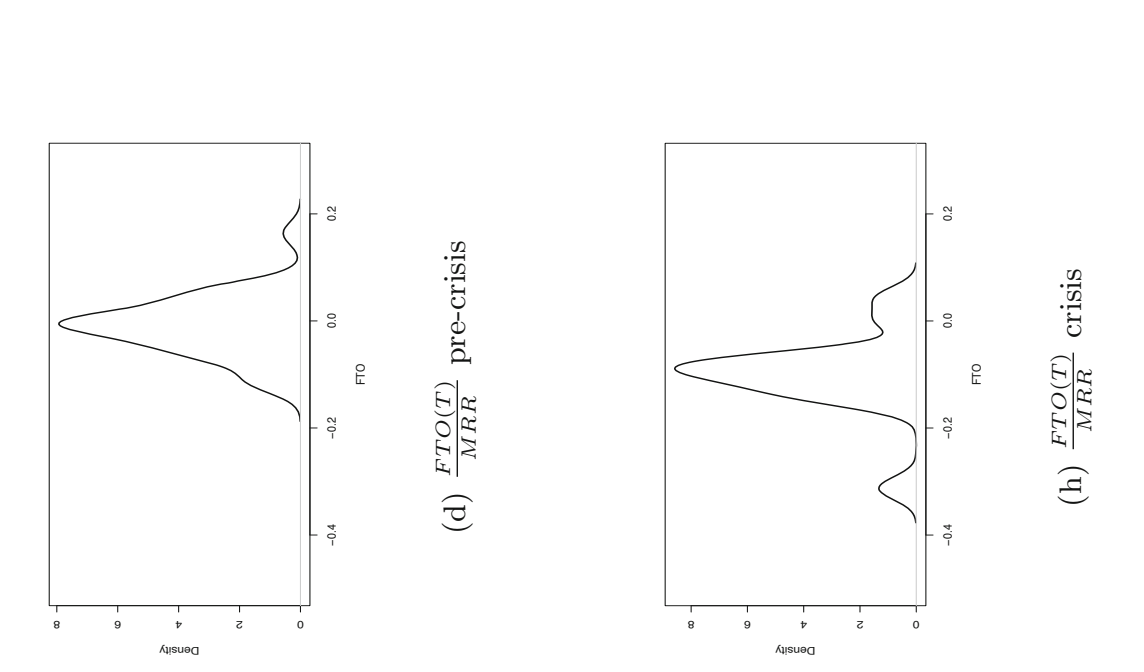

.
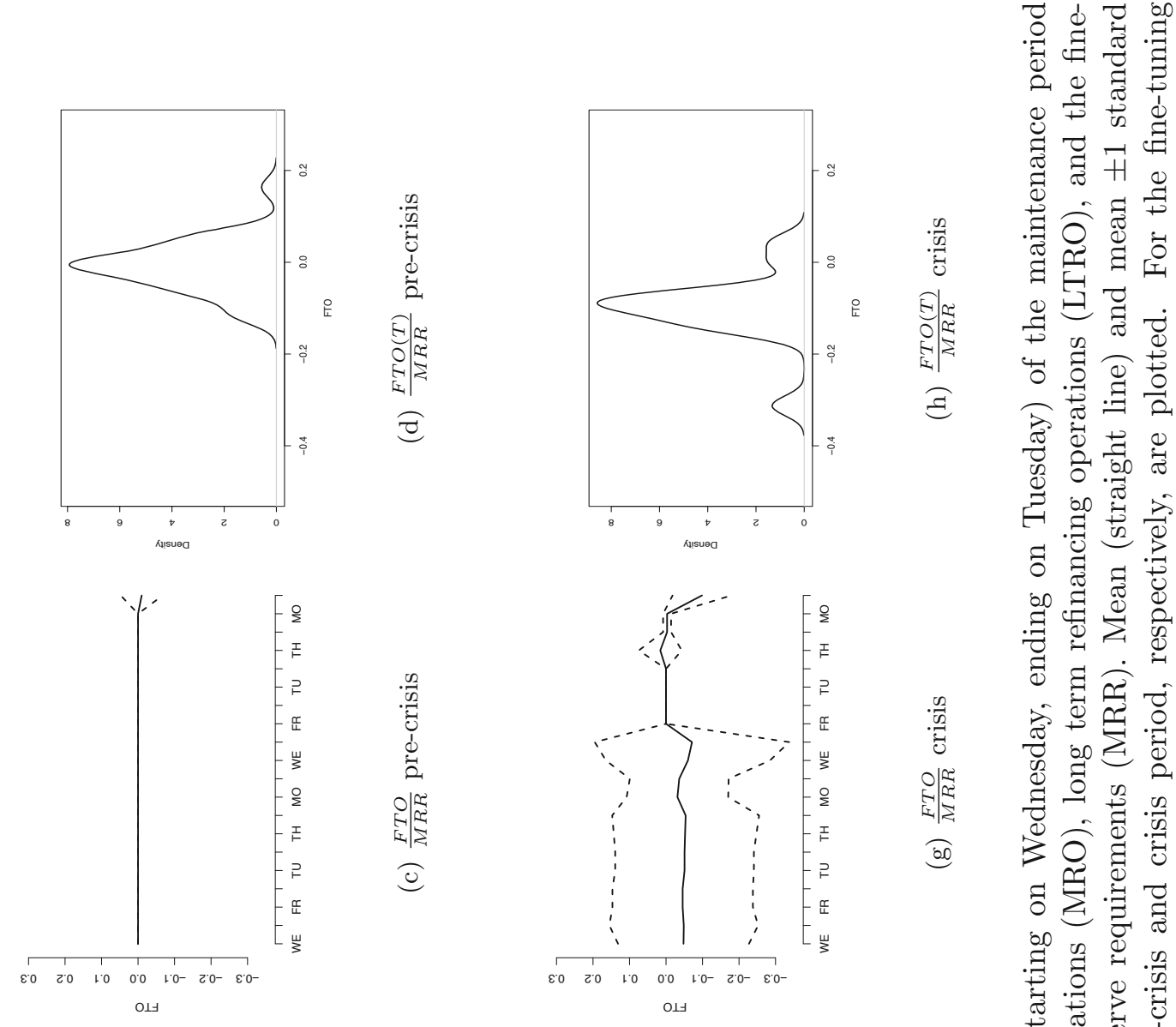

击当范

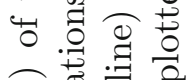

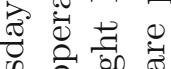

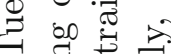

न

䒕 శี

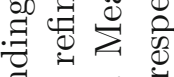

व

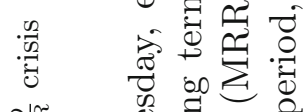

일

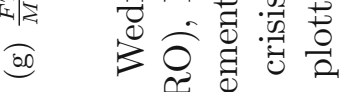

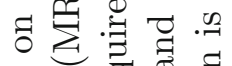

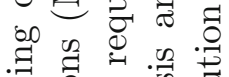

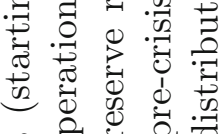

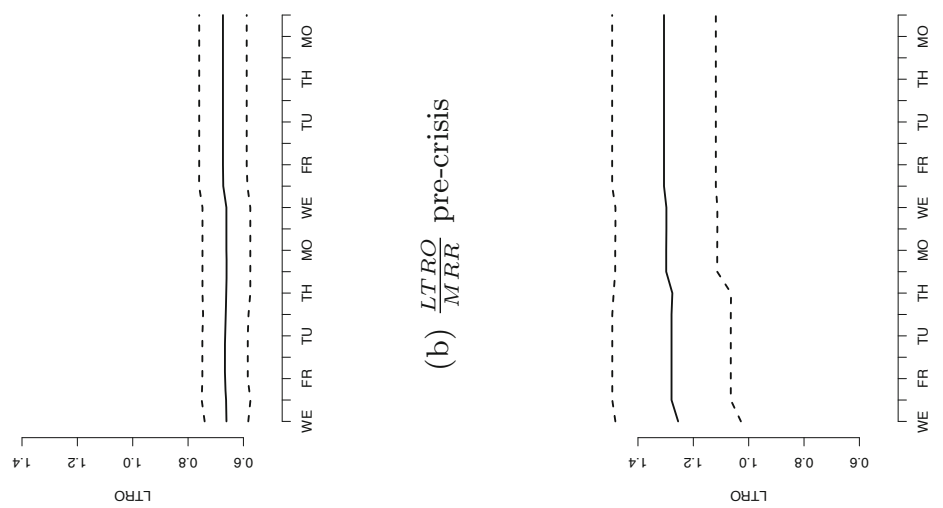

क : مै:

宅

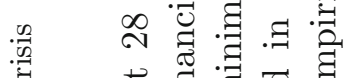

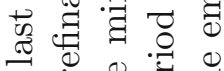

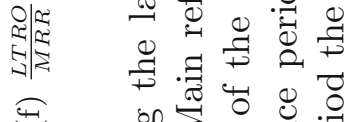

$\Theta$
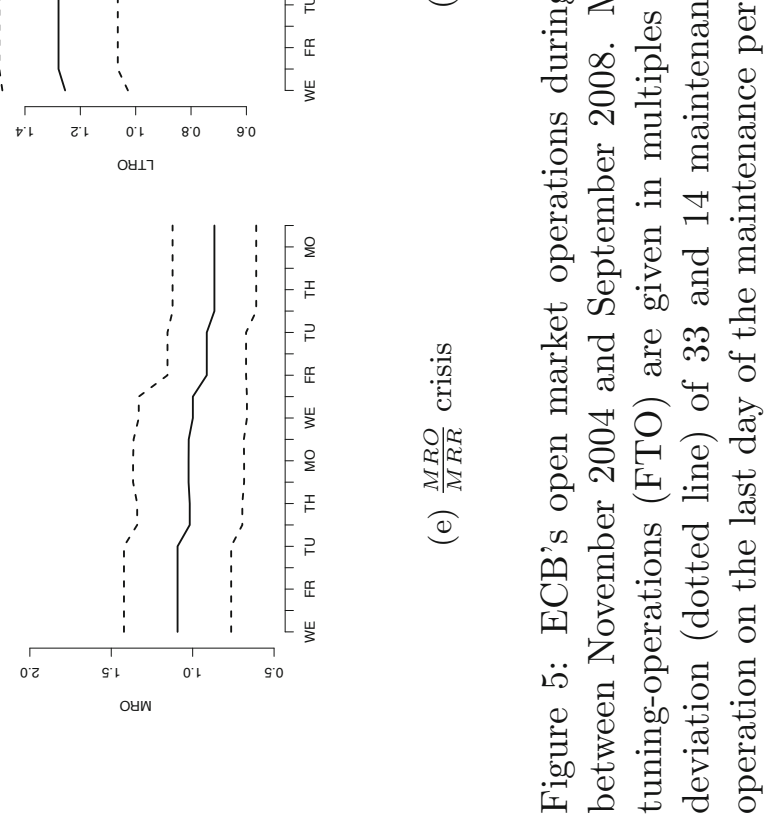

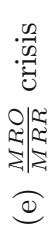



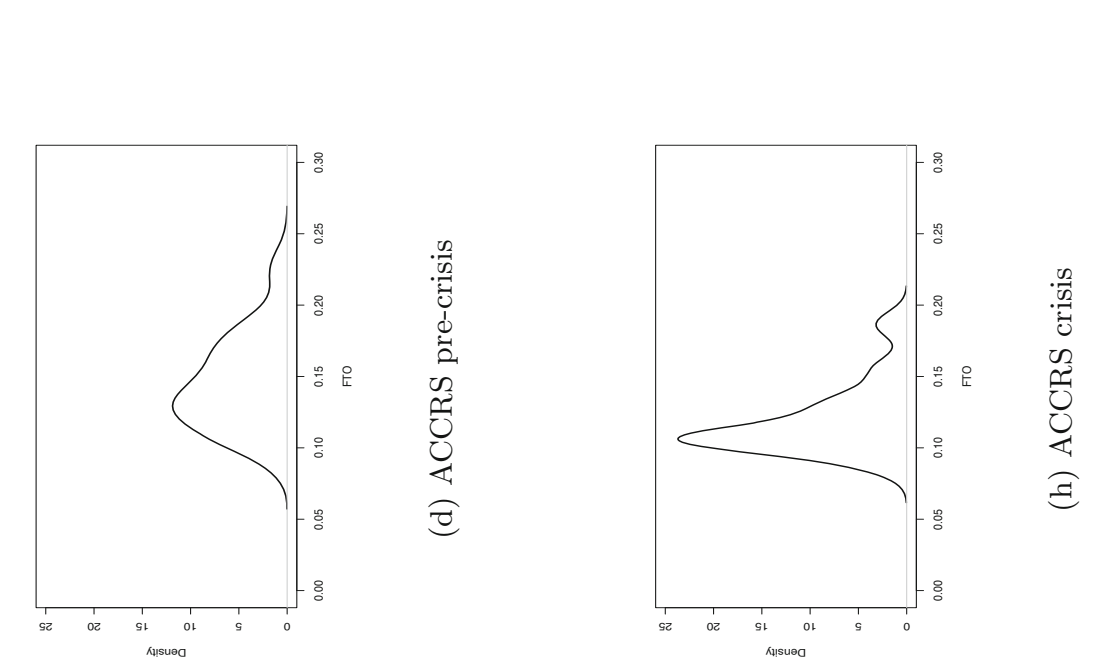

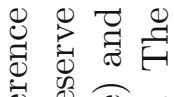

远的

羊目节

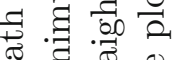

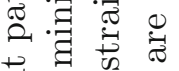

进远

घ 푱

問岁总总

च.

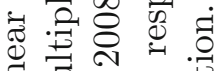

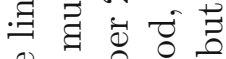

․․ द्व

द

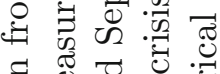

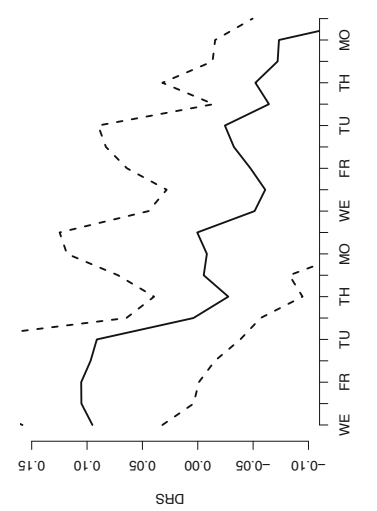

泀

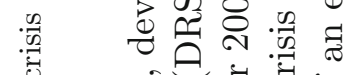

OO

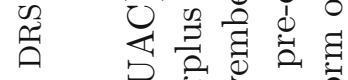

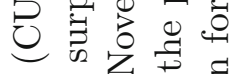

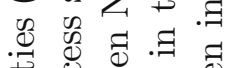

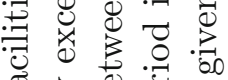

必事造

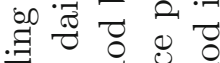

讨

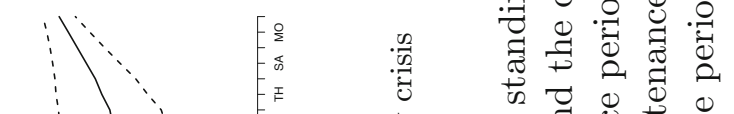

क वै

苟合

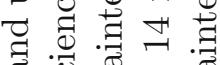

$\pi_{\infty} 0 \mathrm{~d}$

目 8 \%

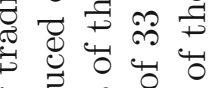

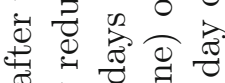

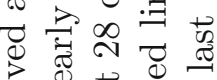

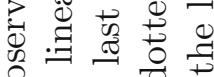

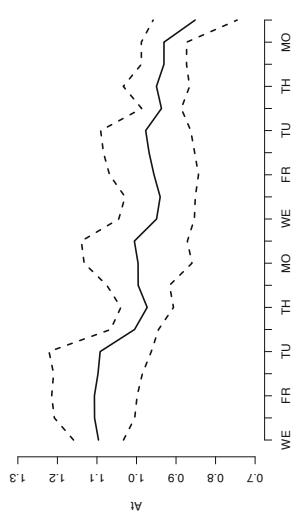

$\therefore$ 웡

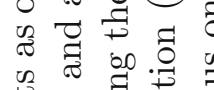

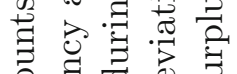

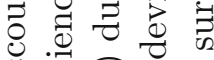

过

원

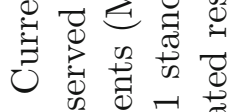

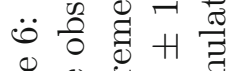

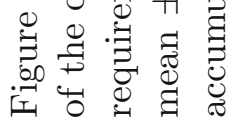




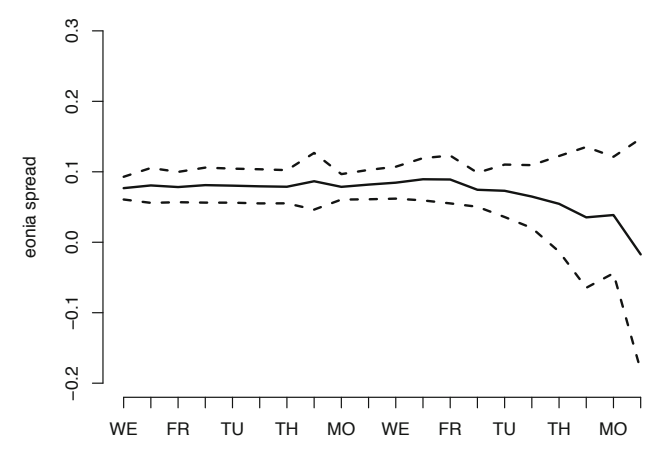

(a) EONIA Spread pre-crisis

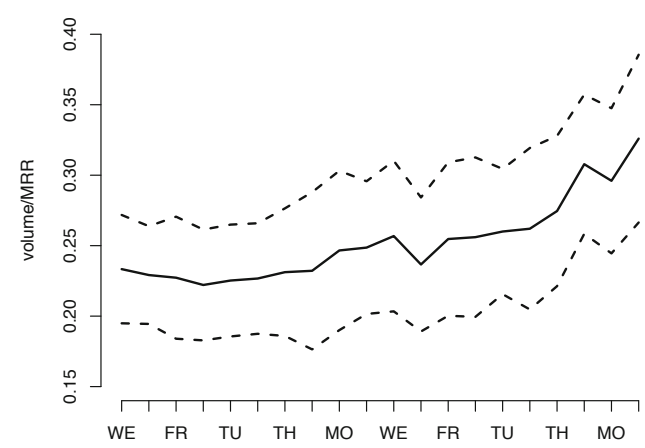

(c) $\frac{\text { Volume }}{M R R}$ pre-crisis

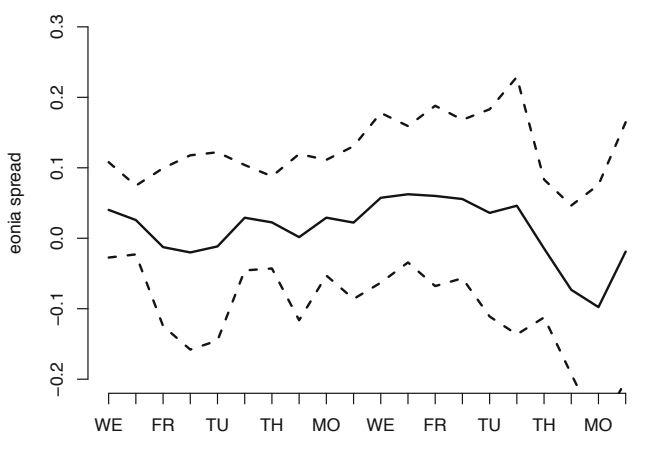

(b) EONIA Spread crisis

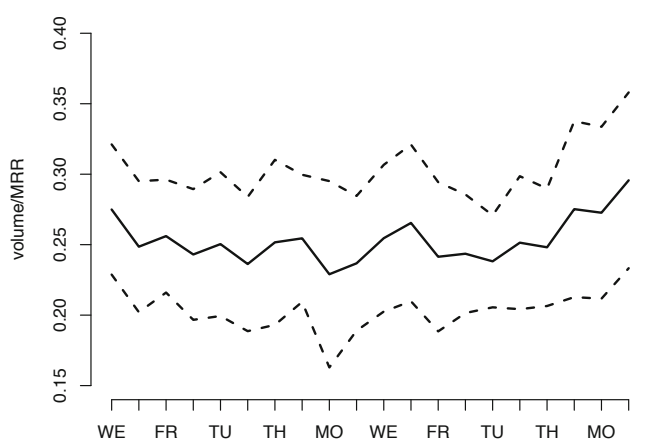

(d) $\frac{\text { Volume }}{M R R}$ crisis

Figure 7: EONIA spread over the minimum bid rate (MBR) and trading volume over the minimum reserve requirements (MRR) during the last 28 days of the maintenance period between November 2004 and September 2008. Mean (straight line) and mean \pm 1 standard deviation (dotted line) of 33 and 14 maintenance period in the pre-crisis and crisis period, respectively, are plotted. 


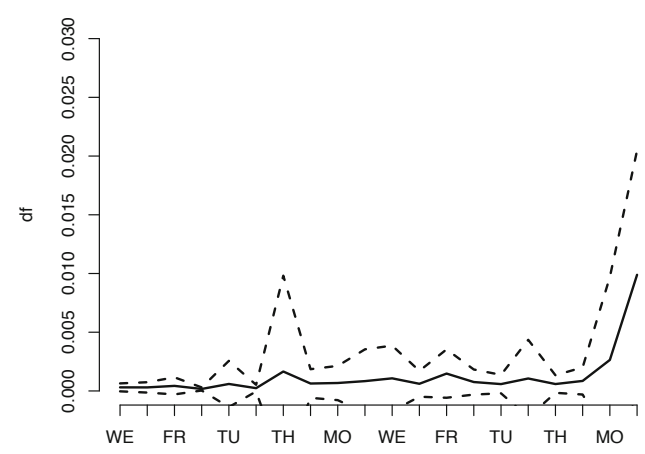

(a) $\frac{D F}{M R R}$ pre-crisis

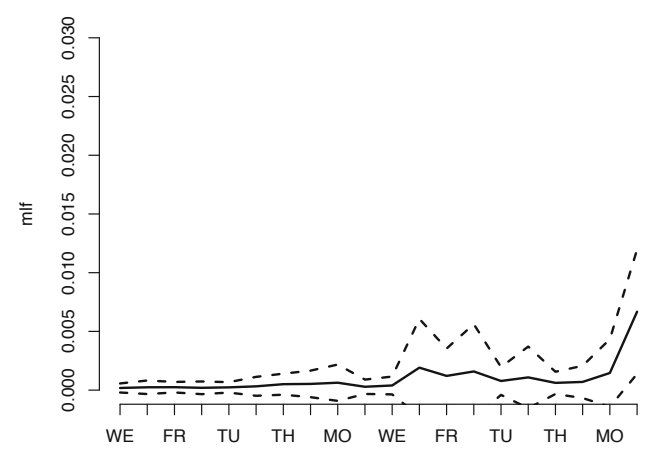

(c) $\frac{M L F}{M R R}$ pre-crisis

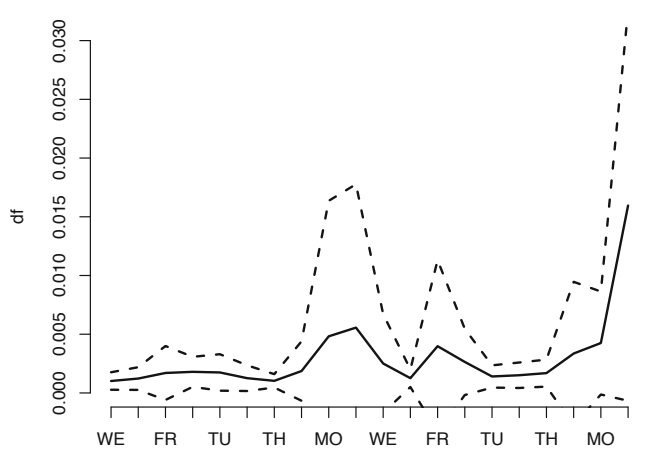

(b) $\frac{D F}{M R R}$ crisis

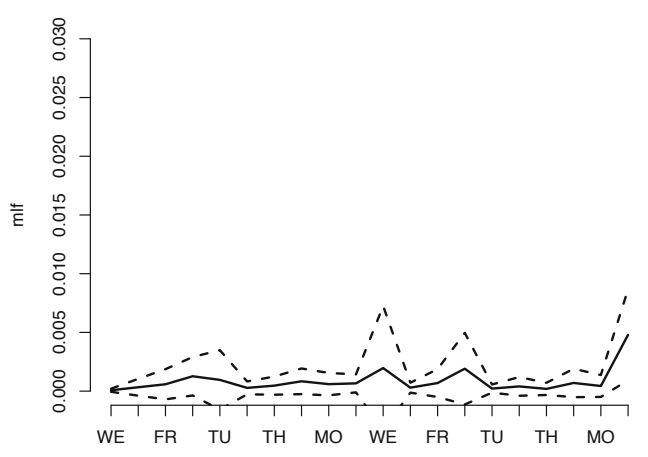

(d) $\frac{M L F}{M R R}$ crisis

Figure 8: Usage of standing facilities (deposit facility (DF) and marginal lending facility (MLF)) in multiples of the minimum reserve requirement (MRR) during the last 28 days of the maintenance period between November 2004 and September 2008. Mean (straight line) and mean \pm 1 standard deviation (dotted line) of 33 and 14 maintenance period in the pre-crisis and crisis period, respectively, are plotted. 

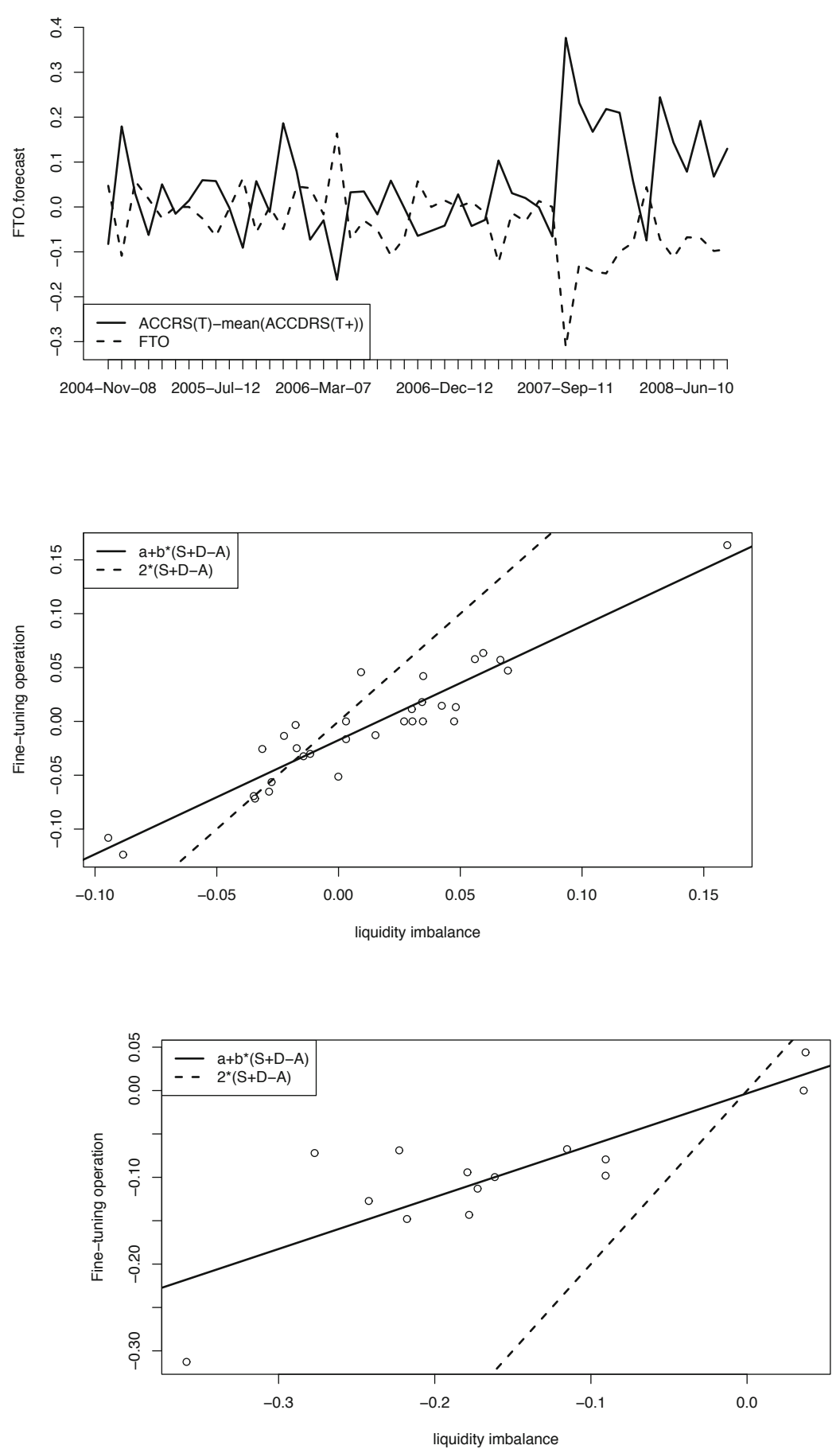

Figure 9: Fine Tuning Operation (FTO) and accumulated reserve surplus on the last day of the maintenance period in the pre-crisis period. The OLS regression gives a $\hat{a}=-0.01741$ $(-0.00326)$ and $\hat{b}=1.05912(0.597753)$ with t-values equal to -4.679 and $14.229(-0.140$ and $4.897)$, respectively. $R^{2}$ is $0.8747(0.6665)$ for the pre-crisis (crisis) period. 
Table 1: Summary statistics cuac $_{t}^{j}$ between March 2003 and July 2007

\begin{tabular}{lrrrrr} 
& Mean & Std. & Skew. & Kurt. & ACF $(1)$ \\
\hline all & & & & & \\
Mean & 100.52 & 52.96 & 2.03 & 21.36 & 0.37 \\
Std. & 2.10 & 25.63 & 2.64 & 52.61 & 0.16 \\
Min. & 95.11 & 0.96 & -0.47 & 2.88 & 0.03 \\
$25 \%$ & 100.00 & 36.62 & 0.77 & 4.83 & 0.26 \\
$50 \%$ & 100.07 & 48.77 & 1.20 & 6.95 & 0.38 \\
$75 \%$ & 100.19 & 65.75 & 1.96 & 12.34 & 0.48 \\
Max. & 113.58 & 153.98 & 17.29 & 407.10 & 0.86 \\
\hline large & & & & & \\
Mean & 99.96 & 51.81 & 1.37 & 11.09 & 0.35 \\
Std. & 0.79 & 18.77 & 1.46 & 23.01 & 0.13 \\
Min. & 95.11 & 22.06 & 0.25 & 2.88 & 0.08 \\
$25 \%$ & 99.99 & 41.49 & 0.74 & 4.62 & 0.25 \\
$50 \%$ & 100.02 & 44.79 & 1.02 & 5.84 & 0.35 \\
$75 \%$ & 100.11 & 64.52 & 1.36 & 7.91 & 0.44 \\
Max. & 101.76 & 105.54 & 9.33 & 156.88 & 0.58 \\
\hline medium & & & & & \\
Mean & 100.68 & 61.81 & 2.62 & 23.62 & 0.33 \\
Std. & 1.26 & 29.55 & 2.06 & 24.81 & 0.17 \\
Min. & 99.12 & 29.98 & -0.47 & 3.98 & 0.03 \\
$25 \%$ & 100.02 & 34.89 & 0.97 & 6.31 & 0.24 \\
$50 \%$ & 100.10 & 57.79 & 1.85 & 11.87 & 0.33 \\
$75 \%$ & 101.07 & 77.42 & 3.50 & 26.34 & 0.45 \\
Max. & 103.56 & 131.71 & 6.94 & 81.87 & 0.67 \\
\hline small & & & & & \\
Mean & 102.52 & 46.42 & 3.89 & 58.77 & 0.54 \\
Std. & 4.34 & 40.19 & 5.09 & 115.70 & 0.18 \\
Min. & 99.65 & 0.96 & -0.39 & 3.12 & 0.13 \\
$25 \%$ & 100.04 & 17.83 & 0.54 & 8.75 & 0.45 \\
$50 \%$ & 100.38 & 48.19 & 2.02 & 11.70 & 0.53 \\
$75 \%$ & 102.98 & 55.77 & 4.45 & 36.20 & 0.64 \\
Max. & 113.58 & 153.98 & 17.29 & 407.10 & 0.86 \\
\hline
\end{tabular}

Estimates are based on individual bank data covering the period March 2003 to July 2007.

Table 2: Kaplan-Meier estimate of survival function $S(\epsilon)_{t}$

\begin{tabular}{|c|c|c|c|c|c|c|c|c|}
\hline & \multicolumn{4}{|c|}{$\epsilon=0$} & \multicolumn{4}{|c|}{$\epsilon=P\left(b_{t}^{j}+a_{t}^{j}\right)$} \\
\hline & all & large & medium & small & all & large & medium & small \\
\hline $\mathrm{t}=\mathrm{T}-4$ & $72.2(0.8)$ & $81.8(0.8)$ & $46.0(1.9)$ & $67.5(2.0)$ & $77.1(0.7)$ & $85.5(0.8)$ & $56.2(1.9)$ & $70.7(2.0)$ \\
\hline $\mathrm{t}=\mathrm{T}-3$ & $65.5(0.8)$ & $74.3(1.0)$ & $40.9(1.9)$ & $61.8(2.1)$ & $70.9(0.8)$ & $78.7(0.9)$ & $51.0(1.9)$ & $65.5(2.0)$ \\
\hline $\mathrm{t}=\mathrm{T}-2$ & $58.2(0.9)$ & $64.6(1.0)$ & $37.6(1.9)$ & $58.7(2.1)$ & $63.9(0.8)$ & $70.3(1.0)$ & $46.9(1.9)$ & $60.5(2.1)$ \\
\hline weekend & $46.8(0.9)$ & $50.0(1.1)$ & $30.0(1.8)$ & $55.2(2.1)$ & $52.9(0.9)$ & $57.1(1.1)$ & $36.9(1.9)$ & $56.6(2.1)$ \\
\hline weekend & $26.8(0.8)$ & $24.5(0.9)$ & $14.5(1.4)$ & $51.1(2.1)$ & $30.6(0.8)$ & $28.6(1.0)$ & $19.9(1.5)$ & $51.5(2.1)$ \\
\hline $\mathrm{t}=\mathrm{T}-1$ & $9.2(0.5)$ & $5.0(0.5)$ & $2.1(0.6)$ & $34.5(2.0)$ & $11.2(0.5)$ & $6.2(0.5)$ & $7.8(1.0)$ & $34.5(2.0)$ \\
\hline $\mathrm{t}=\mathrm{T}$ & $0.4(0.1)$ & $0.4(0.1)$ & $0.3(0.2)$ & $0.4(0.3)$ & $0.8(0.2)$ & $0.7(0.2)$ & $1.3(0.4)$ & $0.6(0.3)$ \\
\hline
\end{tabular}

The survival function $S(\epsilon)_{t}$ gives the probability that up to time t there is no lock-in. The Table plots the last 4 busness days plus the last weekend of the maintenance period for period March 2003 to July 2007. Results are presented for $\epsilon=0$ and $\epsilon$ equal to the probability of an overdraft

$\hat{P}\left(\operatorname{cuac}_{t}^{j}\right) \approx P\left(b_{t}^{j}-a_{t}^{j}\right)$. 


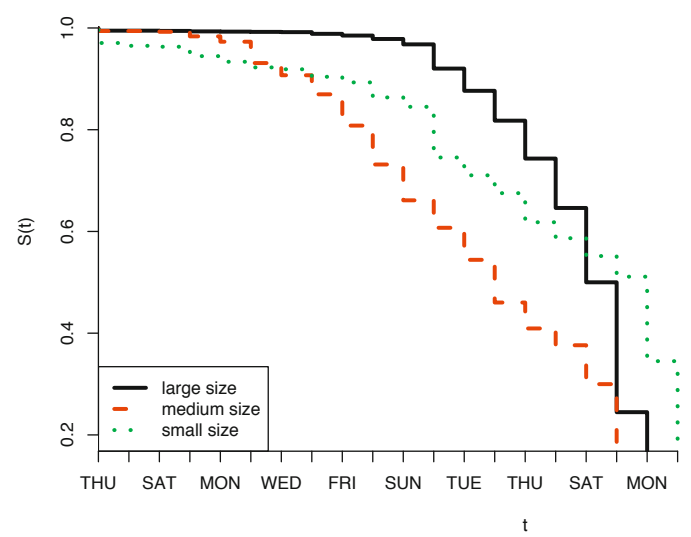

(a) $\epsilon=0$

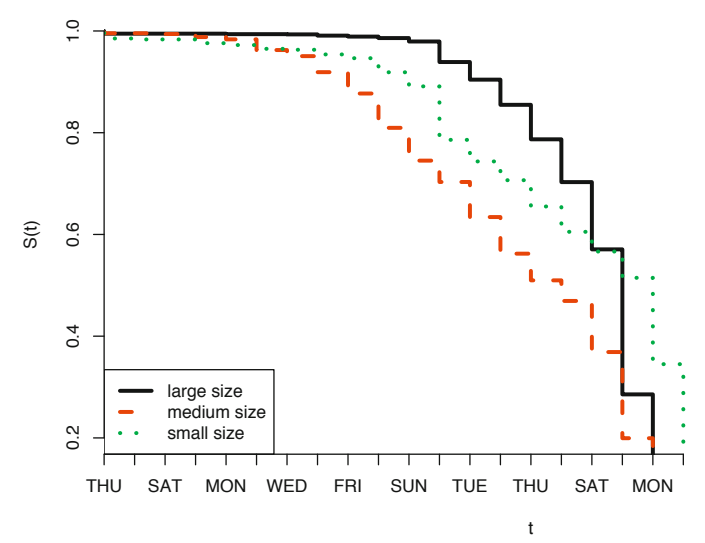

(b) $\epsilon=P\left(b_{t}^{j}-a_{t}^{j}\right)$

Figure 10: Survival function $S(\epsilon)_{t}=\operatorname{Prob}\left(1-P\left(d_{t}^{j}+b_{t}^{j}-a_{t}^{j}\right)<\epsilon\right)$ differentiated according to bank size plotted over the last 20 days of the maintenance period. The survival function $S(\epsilon)_{t}$ gives the average fraction of banks which are not locked-in on day $t$. We consider two thresholds for the regime switch: $\epsilon=0$ and $\epsilon$ equal to the probability of an overdraft $P\left(b_{t}-a_{t}\right)$. Estimates are based on individual banks' current accounts $c u a c_{t}^{j}=a_{t}-b_{t}+\lambda_{t} j$ covering the period March 2003 to July 2007. 
Table 3: Comparison of Euclidean Distance between simulated and observed average EONIA and liquidity characteristics during the last 5 days in the maintenance period.

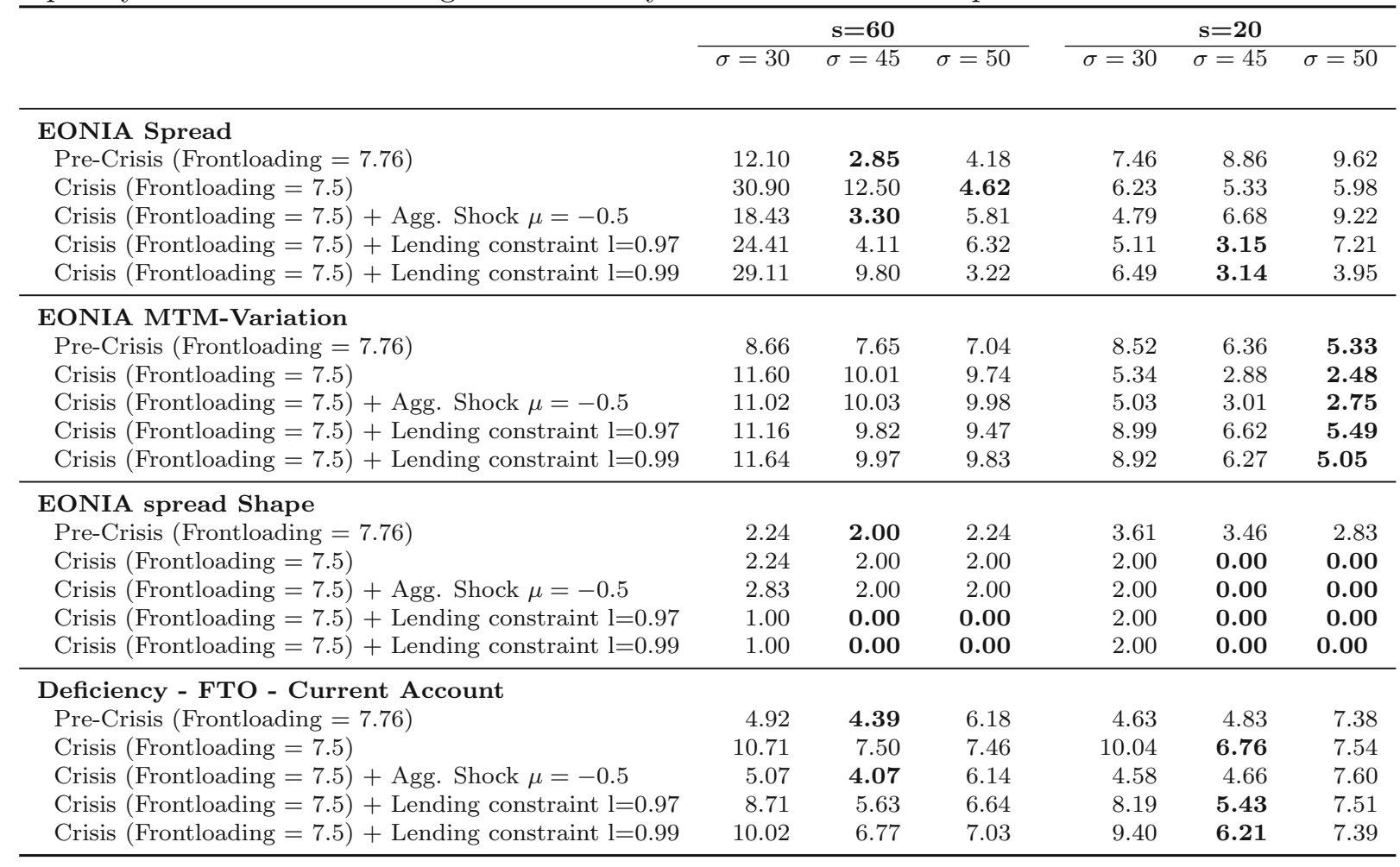

Comparison of observed and simulated EONIA spread, maintainance-period-to-maintenance-period (MTM) variation, EONIA spread shape and liquidity situation for different idiosyncratic shock volatilities $\sigma=\{30,45,50\}$ for different pre-crisis and crisis period scenarios. The pre-crisis period is modeled with frontloading of $F L=7.8$ and without market frictions $(s=60)$ and with market segmentation $s=20$. The crisis period is charactersized by frontloading of $F L=7.5$. The crisis period scenarios consist of combinations of aggregate liquidity shock $\mu=\{-0.5,0\}$, lending constraint $l=\{0.97,0.99,1\}$ and degree of market segmentation $s=\{20,60\}$. For the EONIA spread shape the (unweighted) Euclidian distance of signs of spread changes is calculated. For the other characteristics a weighted Euclidian distance is given which is obtained such that the figures in regime 1 ('WED', 'TUE', 'FRI') have in total a weight of 0.5 and the figures in regime 2 ('MON', 'TUE') also have a weight of 0.5 . The minimum value of a row is plotted in bold face 

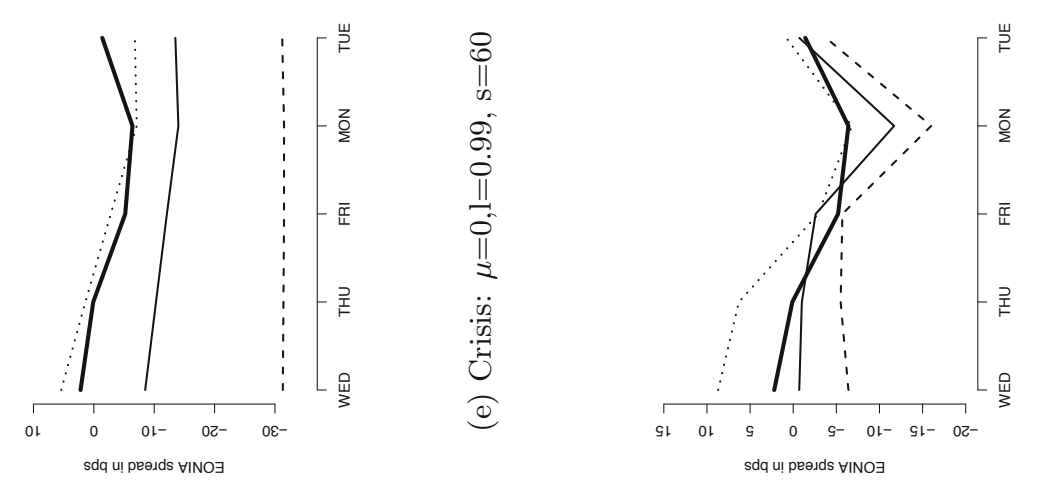

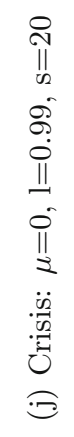

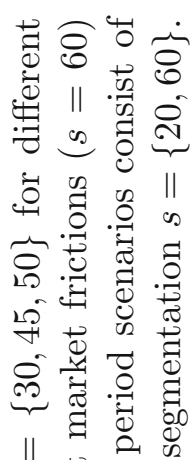

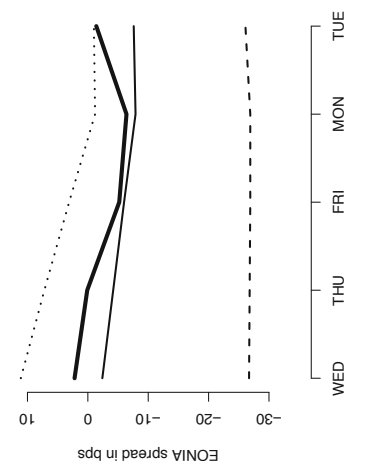

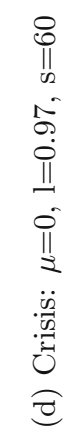
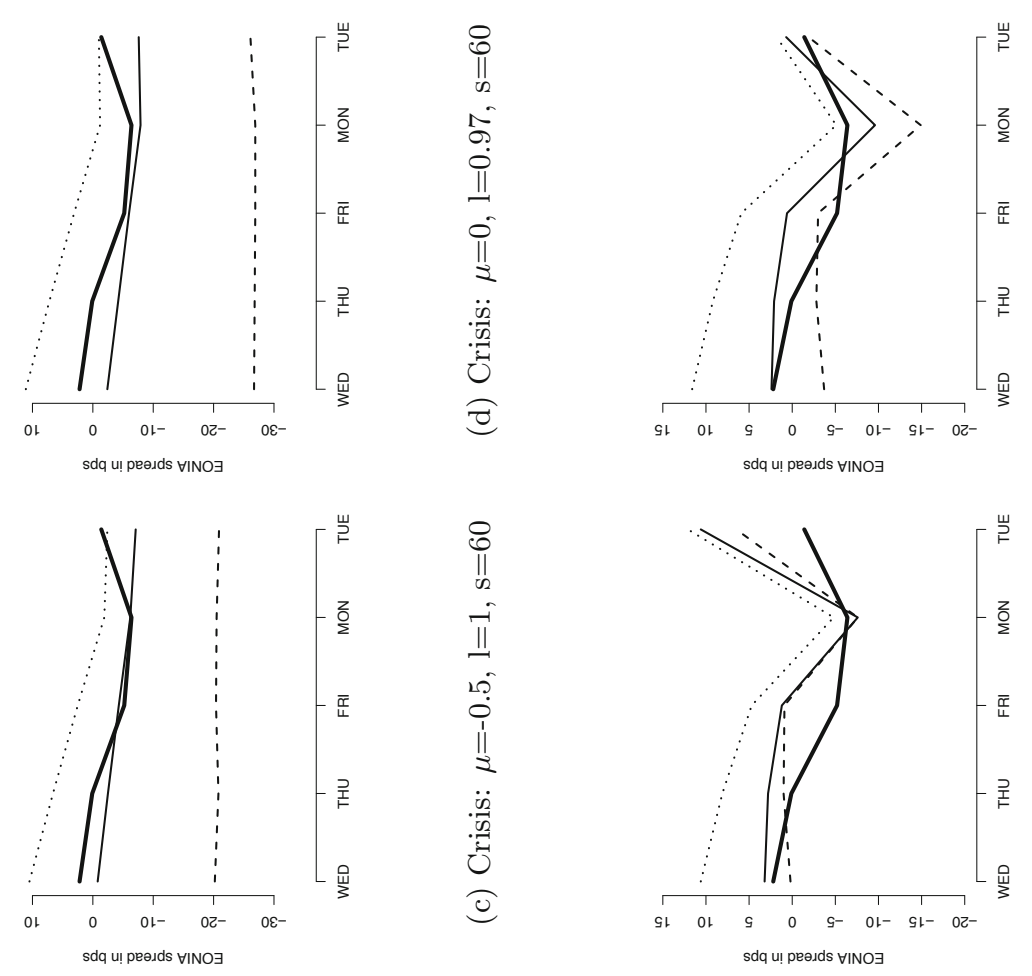

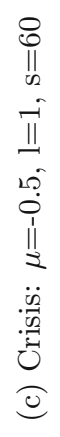
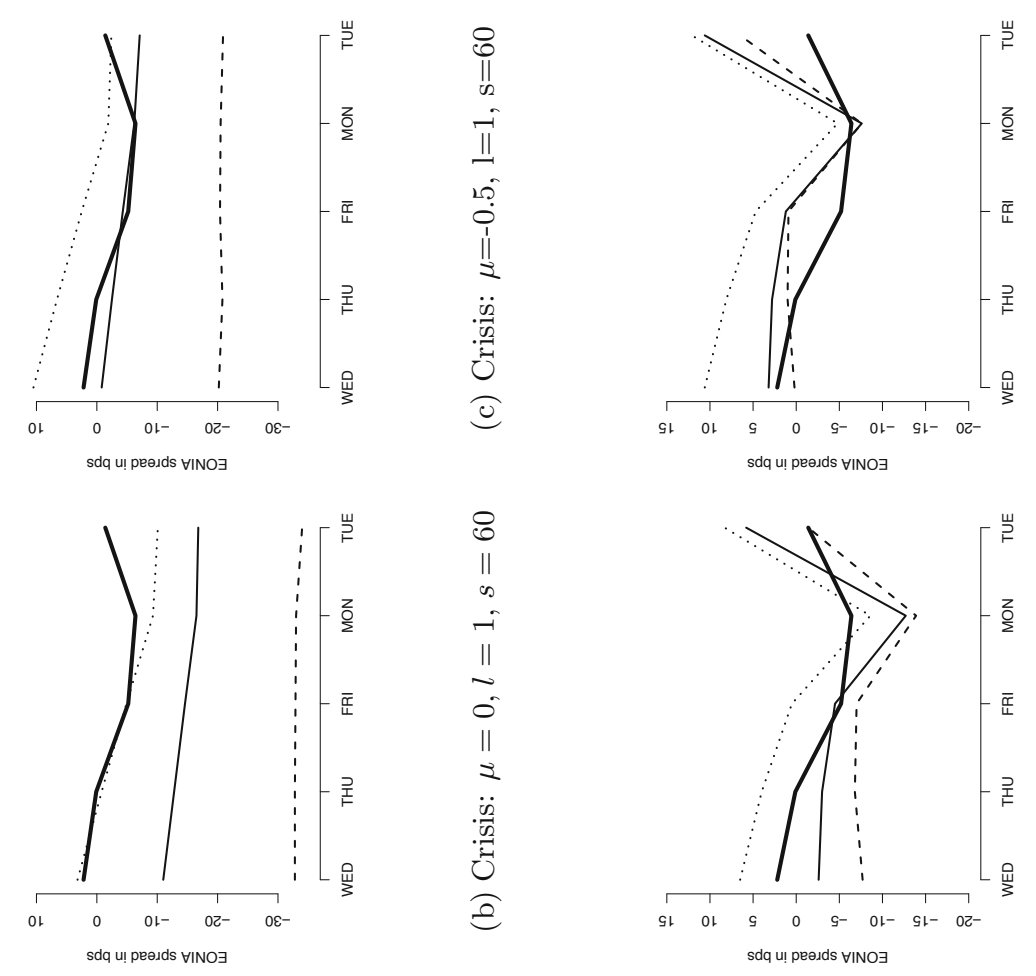

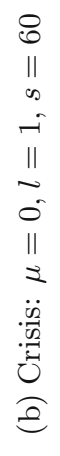

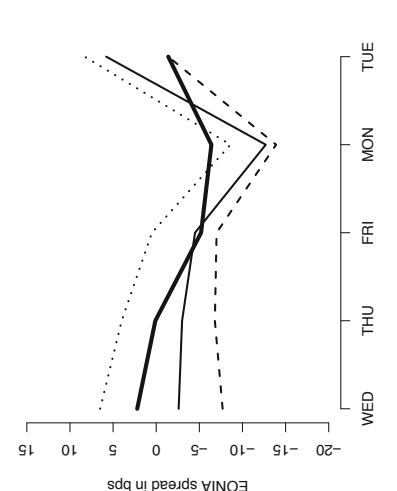

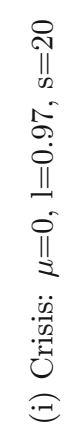

$\begin{array}{lll}11 & 0 \\ 0 & 0 & 0 \\ 0 & 0 & 0\end{array}$

导言芯 寻羊苛

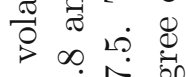
舟

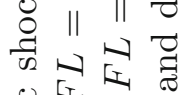

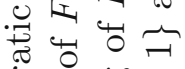

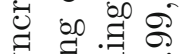

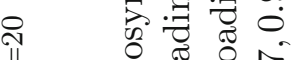

川

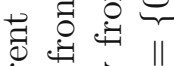
엉

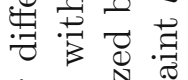

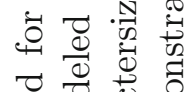
च ত্ర

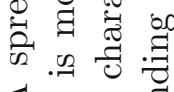

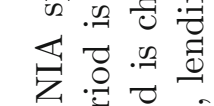
है

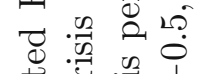
营

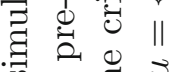
. न 0 ?

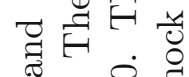
के $\dot{0} \frac{0}{\omega}$ 总.

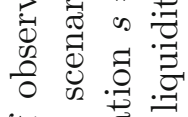
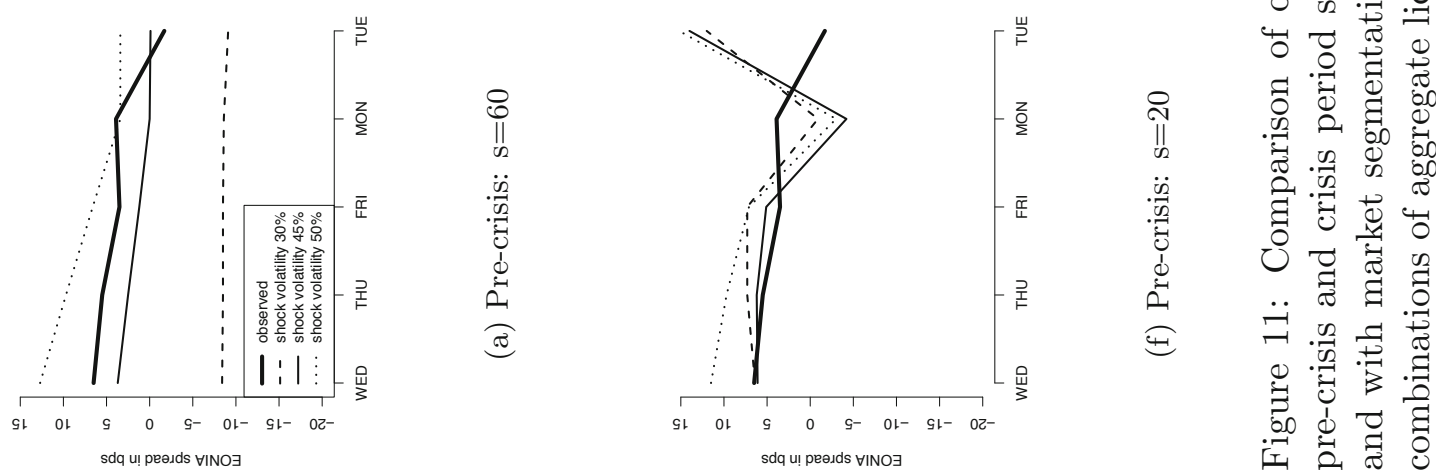

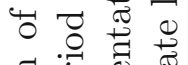


Table 4: Summary statistics $\Delta \overline{c u a c}_{t}^{j}$

\begin{tabular}{|c|c|c|c|c|c|c|}
\hline & Mean & Std. & Skew. & Kurt. & $\begin{array}{r}\text { JB p-val. } \\
(\%)\end{array}$ & $\operatorname{ACF}(1)$ \\
\hline \multicolumn{7}{|l|}{ all } \\
\hline Mean & -0.01 & 40.75 & -0.02 & -0.20 & 0.31 & -0.32 \\
\hline Std. & 1.67 & 18.81 & 0.11 & 0.42 & 0.27 & 0.08 \\
\hline Min. & -3.77 & 6.38 & -0.35 & -0.81 & 0.00 & -0.46 \\
\hline $25 \%$ & -0.81 & 29.64 & -0.08 & -0.42 & 0.08 & -0.38 \\
\hline $50 \%$ & 0.11 & 38.79 & -0.02 & -0.32 & 0.24 & -0.34 \\
\hline $75 \%$ & 0.80 & 50.67 & 0.05 & -0.16 & 0.53 & -0.29 \\
\hline Max. & 6.42 & 96.08 & 0.27 & 1.69 & 0.95 & -0.07 \\
\hline \multicolumn{7}{|l|}{ large } \\
\hline Mean & 0.08 & 42.81 & -0.01 & -0.32 & 0.31 & -0.34 \\
\hline Std. & 1.61 & 16.65 & 0.09 & 0.23 & 0.26 & 0.06 \\
\hline Min. & -3.04 & 12.85 & -0.16 & -0.81 & 0.02 & -0.46 \\
\hline $25 \%$ & -0.81 & 31.28 & -0.08 & -0.44 & 0.10 & -0.37 \\
\hline $50 \%$ & 0.19 & 41.98 & -0.01 & -0.35 & 0.24 & -0.34 \\
\hline $75 \%$ & 0.86 & 51.71 & 0.05 & -0.24 & 0.50 & -0.30 \\
\hline Max. & 6.42 & 96.08 & 0.27 & 0.58 & 0.95 & -0.20 \\
\hline \multicolumn{7}{|c|}{ medium } \\
\hline Mean & -0.21 & 39.95 & -0.00 & -0.03 & 0.26 & -0.32 \\
\hline Std. & 1.70 & 20.09 & 0.13 & 0.61 & 0.25 & 0.08 \\
\hline Min. & -3.77 & 7.60 & -0.34 & -0.47 & 0.00 & -0.44 \\
\hline $25 \%$ & -0.70 & 29.15 & -0.07 & -0.37 & 0.08 & -0.39 \\
\hline $50 \%$ & 0.06 & 37.72 & -0.01 & -0.33 & 0.20 & -0.31 \\
\hline $75 \%$ & 0.66 & 42.52 & 0.06 & -0.06 & 0.32 & -0.28 \\
\hline Max. & 3.04 & 86.01 & 0.20 & 1.69 & 0.70 & -0.16 \\
\hline \multicolumn{7}{|l|}{ small } \\
\hline Mean & -0.12 & 29.71 & -0.12 & 0.15 & 0.41 & -0.25 \\
\hline Std. & 2.11 & 26.54 & 0.13 & 0.60 & 0.37 & 0.12 \\
\hline Min. & -2.57 & 6.38 & -0.35 & -0.57 & 0.00 & -0.39 \\
\hline $25 \%$ & -1.45 & 8.65 & -0.19 & -0.15 & 0.03 & -0.35 \\
\hline $50 \%$ & -0.14 & 18.41 & -0.09 & -0.09 & 0.44 & -0.27 \\
\hline $75 \%$ & 0.72 & 46.02 & -0.03 & 0.32 & 0.70 & -0.17 \\
\hline Max. & 3.92 & 70.70 & 0.04 & 1.31 & 0.90 & -0.07 \\
\hline
\end{tabular}

The residuals are obtained by the time-series regression $\Delta c u a c_{t}^{i}=\alpha+\beta^{M R O} \Delta M R O_{t}+\beta^{L T R O} \Delta L T R O_{t}+\Delta \overline{c u a c}_{t}^{i}$ where $\Delta M R O_{t}, \Delta L T R O_{t}$ and $\Delta c u a c_{t}^{i}$ are the daily absolute changes of the MRO, LTRO and the $i$-th bank end-of-day current account.

Table 5: GMM estimation results based on $\Delta \overline{c u a c}_{t}^{j}$

\begin{tabular}{|c|c|c|c|c|c|c|}
\hline & \multicolumn{3}{|c|}{ no market segmentation $\mathrm{s}=\mathrm{n}$} & \multicolumn{3}{|c|}{ market segmentation $\mathrm{s}=0.1 \mathrm{n}$} \\
\hline & Estimate & Std.Error & $\mathrm{p}$ value & Estimate & $\begin{array}{r}\text { Std.Error } \\
(\%)\end{array}$ & $\mathrm{p}$ value \\
\hline \multicolumn{7}{|l|}{ Parameters } \\
\hline$\sigma_{\lambda, \text { large }}$ & 41.9620 & 8.6418 & 0.0000 & 40.8371 & 7.9070 & 0.0000 \\
\hline$\sigma_{\lambda, \text { medium }}$ & 38.3416 & 9.6320 & 0.0001 & 37.1130 & 8.8864 & 0.0000 \\
\hline$\sigma_{\lambda, \text { small }}$ & 36.1516 & 10.4117 & 0.0005 & 34.8489 & 9.6815 & 0.0003 \\
\hline$\beta$ & 0.4813 & 0.1155 & 0.0000 & 0.5009 & 0.1160 & 0.0000 \\
\hline$\sigma_{\epsilon}$ & 2.9855 & 90.3352 & 0.9736 & 7.1794 & 34.2771 & 0.8341 \\
\hline \multicolumn{7}{|l|}{ J-test } \\
\hline statistic & 0.0011 & & & 0.0017 & & \\
\hline $\mathrm{p}$ value & 0.9735 & & & 0.9676 & & \\
\hline
\end{tabular}




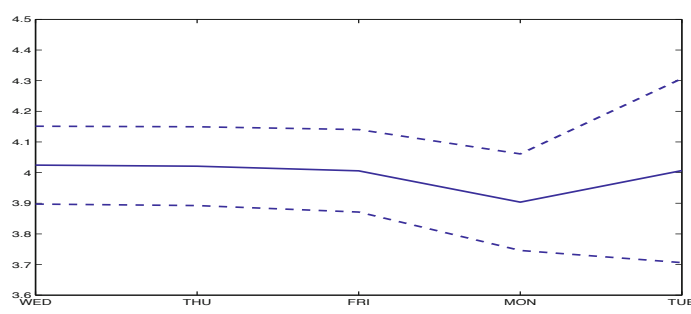

(a) Crisis Obs. Front-loading FTO

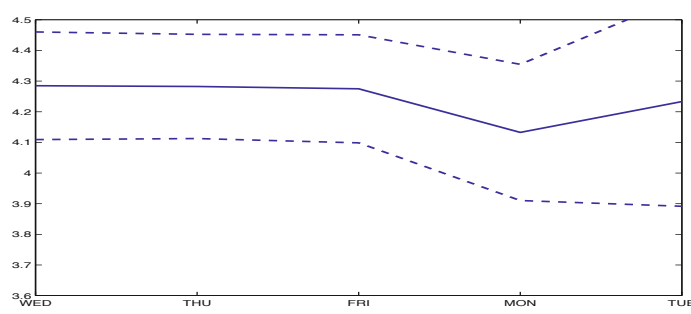

(c) Crisis No Front-loading FTO

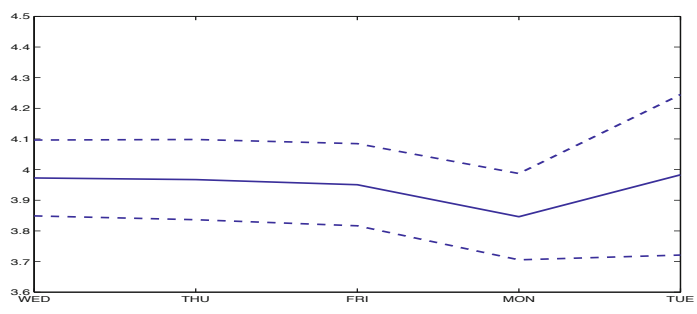

(b) Crisis Obs. Front-loading RB

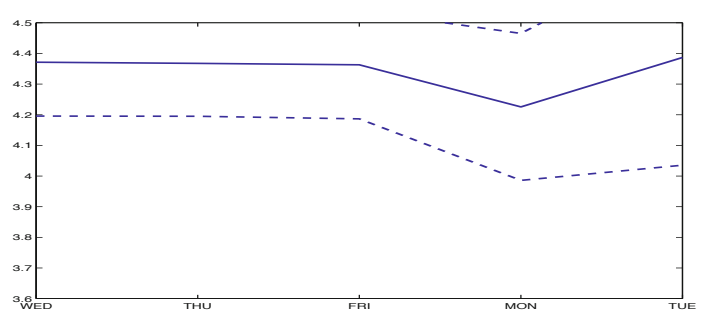

(d) Crisis No Front-loading RB

Figure 12: Comparison of different front-loading policies and end-of-maintenance period policies (fine-tuning operation FTO, reserve band system RB) for a interest rate corridor of $i^{l}-i^{d}=2 \%$. Different scenarios for front-loading policy: high frontloading $\left(D_{T-8}=700\right)$, observed frontloading $\left(D_{T-8}=723.5\right)$, low front-loading $\left(D_{T-8}=750\right)$ and no front-loading $\left(D_{T-8}=807\right)$ are calculated for the crisis period where the starting current accounts were set to $A_{T-8}=96.5$. The lending constraints were assumed to be $l=0.97$ and market segmentation was set to $s=20$. Liquidity shocks were assume to have zero mean $\mu=0$ and a standard deviation of $\sigma=45$. The fine-tuning-operation (FTO) policy was assumed to target an aggregate surplus of $S=12$ $\left(f=S+D_{T}-A_{T}\right)$. The reserve band policy assume a reserve band of $r b=28$. 
Table 6: Policy Comparison based on simulations of 1000 maintenance periods

\begin{tabular}{|c|c|c|c|c|c|c|}
\hline & \multicolumn{2}{|c|}{$i_{T}-M B R$} & \multicolumn{2}{|c|}{ St.dev $i_{t}$} & \multicolumn{2}{|c|}{ Bid-ask Spread } \\
\hline & Avg. & Diff. & Avg. & Diff. & Avg. & Diff. \\
\hline Obs. Frontloading and FTO & 0.7 & & $10.4^{* * *}$ & & $2.7^{* * *}$ & \\
\hline Obs. Frontloading and RB & -1.7 & $-2.4^{* * *}$ & $9.4^{* * *}$ & $-1.0 * * *$ & $2.6^{* * *}$ & $-0.1 * * *$ \\
\hline No Frontloading and FTO & $23.3^{* * *}$ & $22.7 * * *$ & $11.2^{* * *}$ & $0.8 * *$ & $3.3^{* * *}$ & $0.7 * * *$ \\
\hline \multirow[t]{3}{*}{ No Frontloading and RB } & $38.7^{* * *}$ & $38.0^{* * *}$ & $11.7^{* * *}$ & $1.3 * * *$ & $4.0 * * *$ & $1.2^{* * *}$ \\
\hline & \multicolumn{2}{|c|}{ Tot. Liq. Prov. } & \multicolumn{2}{|c|}{ Liq. Prov. $\mathrm{t}=\mathrm{T}$} & \multicolumn{2}{|c|}{ Avg. Trading } \\
\hline & Avg. & Diff. & Avg. & Diff. & Avg. & Diff. \\
\hline Obs. Frontloading and FTO & $45.5^{* * *}$ & & $-3.0 * * *$ & & $113.2^{* * *}$ & \\
\hline Obs. Frontloading and RB & $45.2^{* * *}$ & $-0.3 * * *$ & $-3.3 * * *$ & $-0.3 * * *$ & $114.1^{* * *}$ & $0.9^{* * *}$ \\
\hline No Frontloading and FTO & $61.0 * * *$ & $15.4^{* * *}$ & $61.0 * * *$ & $64.0 * * *$ & $109.1^{* * *}$ & $-4.1^{* * *}$ \\
\hline No Frontloading and RB & $61.0^{* * *}$ & $15.7 * * *$ & $61.0 * * *$ & $63.8 * * *$ & $104.2^{* * *}$ & $-9.0 * * *$ \\
\hline
\end{tabular}

Simulation of 1000 maintenance periods for comparison of open market operation policies. Quality criteria: average EONIA spread $i_{T}-M B R$, the bid-ask $\operatorname{spread}_{T}$ due to lending constraints and the time-series standard deviation of the EONIA over the last 5 days is given in bps; liquidity provision 'Tot.Liq.Prov' is calculated as amount of front-loading plus liquidity absorbtion or provision on $t=T$ ('Liq.Prov $\mathrm{t}=\mathrm{T}$ ') as percentage of the minimum reserve requirements (MRR); the average trading volume is calculated over the last two days in regime II (as the trading volume is the same for all policies in regime I) and also given in percent of the MRR. Two different scenarios for the ECB's frontloading policy are considered: observed frontloading $\left(D_{T-8}=723.5\right)$ and no front-loading $\left.\left(D_{T-8}=807\right)\right)$ are calculated for the crisis period where the starting values for current accounts were set to $A_{T-8}=96.5$. The lending constraints were assumed to be $l=0.97$ and market segmentation was set to $s=20$. Liquidity shocks were assume to have zero mean $\mu=0$ and a standard deviation of $\sigma=45$. The fine-tuning-operation (FTO) policy was assumed to target an aggregate surplus of $S=12$. The reserve band policy $(\mathrm{RB})$ assume a reserve band of $r b=28$. For each quality measure the average ('Avg.') over 1000 simulation and the difference ('Diff.') of each policy to the policy of 'Obs. Frontloading and FTO' is reported. For each reported figure we test for significant difference from zero: $* * * 1 \%, * * 5 \%$ significance level. 
